Annuaire suisse de politique de développement

3 | 1983

Annuaire Suisse - Tiers Monde 1983

\title{
L'aide au développement des communes et des cantons Suisses
}

\section{Eric Hoesli}

\section{(2) OpenEdition}

1 Journals

Édition électronique

URL : http://journals.openedition.org/aspd/1012

DOI : 10.4000/aspd.1012

ISSN : 1663-9669

Éditeur

Institut de hautes études internationales et du développement

\section{Édition imprimée}

Date de publication : 1 janvier 1983

Pagination : 155-194

ISSN : 1660-5934

\section{Référence électronique}

Eric Hoesli, «L'aide au développement des communes et des cantons Suisses », Annuaire suisse de politique de développement [En ligne], 3 | 1983, mis en ligne le 28 novembre 2012, consulté le 08 septembre 2020. URL : http://journals.openedition.org/aspd/1012 ; DOI : https://doi.org/10.4000/ aspd. 1012 


\title{
L'AIDE AU DEVELOPPEMENT DES COMMUNES ET DES CANTONS SUISSES
}

\author{
Eric Hoesli
}

\begin{abstract}
Zusammenfassung: Die Entwicklungshilfe der schweizerischen Gemeinden und Kantone
\end{abstract}

Wie aus den von der DEH erstellten Statistiken hervorgeht, haben die Gemeinden und Kantone 1982 die - im Vergleich zu den vom Bund aufgewendeten 480 Millionen - bescheidene Summe von 7,5 Mio Franken für die Entwicklungs- und humanitäre Hilfe ausgegeben. Allerdings zeigt dies eine positive Tendenz innerhalb der öffentlichen Meinung auf. Im Sinne des entsprechenden Bundesgesetzes lässt der Bund den Gemeinden und Kantonen im Bereich der Entwicklungshilfe einen grossen Spielraum, obwohl besonders die DEH den in diesem Bereich weniger versierten Gemeinden und Kantonen - wenn nötig - assistiert. Im Gegensatz zur humanitären Hilfe wird die von letzteren geleistete Entwicklungshilfe jedoch mehr von der politischen Orientierung der Empfängerländer abhängig gemacht. Sowohl die humanitäre Hilfe als auch die mit mehr Vielfalt ausgestattete Entwicklungshilfe werden als indirekte Hilfe über eine spezialisierte Entwicklungshilfeorganisation befördert oder von den $\mathrm{Ge}$ meinden (einschliesslich der Kirchen) und Kantonen direkt geplant und verwaltet. Obwohl die gegenwärtige Wirtschaftskrise die Gefahr einer Kürzung der Entwicklungshilfe in sich birgt, sollte die von den Gemeinden und Kantonen geleistete Entwicklungshilfe weiterhin unterstützt werden.

\section{ELEMENTS D'UNE DEFINITION}

En 1982, l'aide au développement apportée par les communes suisses dépassait 4 millions de francs. Celle des cantons approchait du cap des 3,5 millions. Au total, c'est plus de 7,5 millions de francs que les collectivités publiques autres que la Confédération consacraient à la coopération au développement et à l'aide humanitaire.

Appréciable en lui-même, ce montant est pourtant faible si on le compare aux prestations de la Confédération (480 millions) ou même au total de l'aide privée suisse (103 millions) la même année. II s'agit cependant de considérer avec attention les efforts entrepris par les communes et les cantons avant de les juger quantité négligeable. Car, 
à l'ombre de ces chiffres modestes, la réalité de l'aide communale et cantonale au développement révèle une richesse et une complexité peu communes. Elle est souvent l'expression d'une volonté d'ouverture et de solidarité de la part d'autorités locales ou même de citoyens qui tentent de matérialiser leur engagement à l'échelon politique qui leur est le plus proche, le seul qu'ils peuvent réellement appréhender.

En ce sens, l'aide des communes et des cantons suisses est aussi un indicateur précieux des tendances de l'opinion publique; elle révèle clairement le chemin déjà parcouru et les obstacles qu'il reste encore à surmonter pour convaincre la population de la nécessité pour la Suisse de coopérer avec ses nouveaux partenaires des pays en développement.

Avant de plonger plus profondément dans la réalité complexe des liens tissés entre nos communes, nos cantons et les Etats du Tiers Monde, il convient de faire quelques mises au point.

Dans la statistique réalisée chaque année par Swissaid (1), les prestations des communes et des cantons figurent au chapitre de l'aide privée. Cela est dû essentiellement aux mécanismes de la coopération cantonale et communale. Comme nous le verrons plus loin, les collectivités publiques ne gèrent que rarement elles-mêmes les sommes qu'elles affectent à l'aide au développement. Dans la plupart des cas, elles préfèrent s'en remettre aux organisations privées de développement (dites aussi CEuvres d'entraide) qui sont compétentes dans ce domaine, et qui restent d'ailleurs partiellement sous le contrôle de la Confédération.

Les organisations privées qui récoltent les fonds des communes et des cantons, les comptabilisent donc dans leurs recettes. Ainsi, les CEuvres d'entraide ont annoncé que le montant total de l'aide privée suisse s'élevait à 103 millions de francs, y compris les contributions communales et cantonales.

La Direction de la Coopération au développement et de l'Aide humanitaire (DDA), l'organisme fédéral chargé de la conception, de la gestion et du contrôle de l'aide suisse, intègre quant à elle les contributions cantonales et communales dans le total de l'aide publique suisse au développement (APD) et les soustrait au total de l'aide privée. Cela afin de se conformer aux normes établies sur le plan international. Chaque année, en effet, les 17 pays occidentaux membres du Comité d'aide au développement de I'OCDE (CAD) se réunissent pour comparer leurs efforts respectifs. Or, selon les règles du CAD, "seuls sont considérés comme "aide publique au développement" (APD) les apports (dons ou prêts):

- accordés par le secteur public

- dans le but essentiel d'améliorer le développement économique et le niveau de vie

- assortis de conditions financières libérales» (2). 
II ne fait aucun doute que les montants affectés par les communes et les cantons suisses aux pays en développement répondent parfaitement à toutes ces conditions. A dire vrai, les critères utilisés par Swissaid sont même plus exigeants puisqu'ils ne tiennent compte que des "contributions versées dans le cadre de la coopération au développement et de l'aide humanitaire sous forme de dons et dont le but désintéressé est de surmonter des situations économiques et sociales difficiles dans une approche à long terme (coopération au développement) ou de soulager à court terme des cas de détresse engendrés par des catastrophes naturelles, des conflits armés, des déplacements de réfugiés, etc. (aide humanitaire). Sont de ce fait exclus les transferts à caractère commercial et les prêts $[. .$.$] , ainsi que les dépenses liées à$ des activités missionnaires au sens strict, telles que formation des prêtres, publications religieuses, constructions d'églises, etc.» (3).

La divergence statistique entre la DDA et les organismes privés n'a d'ailleurs pas grande portée du moment que le montant de l'aide communale et cantonale $(7,5$ millions) ne représente que $1,4 \%$ de l'aide publique au développement (1982).

II faut éviter aussi de confondre I'aide communale et cantonale au développement avec les dons de ces collectivités à l'étranger. En effet, les communes et les cantons affectent parfois des sommes considérables à des nations qui ne sont pas à proprement parler des pays en développement.

La commune de la Chaux-de-Fonds porte chaque année à son budget un montant de 100.000 francs qui permet à son exécutif de répondre à des demandes d'aide lorsqu'il l'estime justifié. Au cours de ces derniers mois la commune a ainsi donné une suite favorable à des requêtes concernant la Pologne, le Nigeria, le Liban et la Sicile. Elle a aussi soutenu financièrement les familles de détenus politiques d'un village turc, en permettant en outre à un observateur d'aller suivre le déroulement du procès en Turquie.

Au total, la Chaux-de-Fonds a déboursé 41.000 francs au titre de l'aide humanitaire dans les seuls pays en développement durant l'année 1982.

II n'existe pas à notre connaissance de statistique dressant un bilan de l'ensemble des dons à l'étranger effectués par les communes et les cantons suisses. Mais l'on peut tenir pour probable que le nombre de collectivités qui versent de l'argent lors d'événements politiques ou de cataclysmes naturels survenus en Europe est plus élevé que celui des communes et cantons qui sont actifs dans le domaine de l'aide au développement.

Plusieurs raisons à cela. Tout d'abord la proximité de l'événement provoque une plus grande émotion au sein de la population locale, y compris au niveau des autorités. Le tremblement de terre du Frioul puis celui du sud de la péninsule italienne en 1981 ont provoqué un très vif émoi dans toute la Suisse et ont poussé de très nombreuses commu- 
nes à offrir une assistance financière. De même lors des récents événements de Pologne où plusieurs collectes avaient été organisées pour venir en aide à la population, notamment par le biais de l'aide alimentaire.

D'autres facteurs expliquent aussi la retenue dont font preuve bon nombre de communes lorsqu'il s'agit d'aide destinée spécifiquement aux pays en développement. La coopération est souvent l'objet de méfiance. La population a trop souvent entendu parler $d^{\prime}$ argent détourné, de gaspillages ou d'utilisations contre-performantes au cours des années passées. L'image des fameux chasse-neige livrés en Afrique ou des millions empochés par le dictateur Somoza au Nicaragua après le tremblement de terre revient dans de nombreuses conversations. Les clichés ont la vie dure. Et ils représentent des obstacles non négligeables lorsqu'il s'agit de convaincre un législatif communal ou cantonal de l'efficacité de la coopération au développement. La tâche est encore plus rude lorsque le projet est soumis au corps électoral dans son entier.

Le 5 mars 1972 les citoyens de la commune de Zurich étaient appelés à se prononcer sur un projet d'arrêté communal donnant la compétence au législatif (Gemeinderat) et à l'exécutif (Stadtrat) de soutenir chaque année des projets de développement jusqu'à concurrence d'un montant équivalant à $1 \%$ des revenus fiscaux de l'exercice écoulé. Le projet fut approuvé en votation populaire à une très faible majorité.

La raison de ce succès tenait sans doute au fait que le projet prévoyait d'apporter simultanément une aide aux régions les plus défavorisées du globe, situées dans les pays en développement et aux communes les plus pauvres de Suisse. L'arrêté coupait l'herbe sous les pieds des détracteurs de l'aide au développement qui affirment que la priorité doit être accordée à ceux de nos concitoyens qui ont encore besoin d'être soutenus.

Fort de l'approbation du peuple, le Conseil communal zurichois a déboursé chaque année depuis 1972 un demi-million de francs en faveur de la coopération au développement et un autre demi-million destiné aux petites communes de haute montagne de notre pays.

La prudence des autorités zurichoises ne s'était d'ailleurs pas arrêtée là. Soucieuses d'augmenter encore les chances de l'arrêté communal soumis au vote, elles avaient joint une dernière phrase au texte de l'arrêté: "une partie du crédit d'aide au développement à l'étranger peut soutenir des projets dont les Suisses de l'étranger dans le besoin seraient les premiers bénéficiaires» (trad.).

On imagine aisément l'impact psychologique que peut avoir une telle formule sur l'électeur moyen. Chacun est à même de s'identifier avec un concitoyen en difficultés à l'autre bout du monde, comme le démontrent d'ailleurs constamment les journaux helvétiques qui font à chaque catastrophe un minutieux décompte des morts possédant un passeport à croix blanche.

Cette astuce juridique est-elle à l'origine du succès rencontré lors de la votation de 1972 ? II est naturellement impossible de l'affirmer. On constate en tout cas que pas un centime des quelque 5 millions versés au titre de l'aide au 
développement entre 1972 et 1981 n'a servi à financer un projet dont les bénéficiaires auraient été des Suisses de l'étranger.

Comme nous venons de le montrer, toute l'aide que les communes et cantons apportent à l'étranger n'est donc pas de l'aide publique au développement. Mais il faut aller plus loin encore: certains projets de développement peuvent être financés par des collectivités publiques sans que ces dernières apparaissent pour autant dans la liste des communes donatrices.

En 1981, des fonds prélevés sur les impôts communaux lucernois ont permis d'équiper un hôpital à Puthiamputur (Inde), de construire un pont sur le Mbombo (Zaïre), de financer un programme de lutte contre la faim à Talibura (Indonésie), d'édifier un poste sanitaire à Encarnacion (Paraguay), etc... Le tout pour un montant de 187.000 francs. Le niveau élevé de cette aide placerait Lucerne au quatrième rang des prestations communales... si elle avait été comptabilisée parmi les contributions publiques. Or Lucerne ne figure pas sur la liste des communes donatrices.

La même année les finances communales saint-galloises ont donné 26.300 francs à divers projets d'adduction d'eau, de soins de santé primaire et de travail social en Inde, en Tanzanie et à Madagascar. Là non plus, pas trace de contribution communale dans la statistique officielle.

Les registres fédéraux seraient-ils mal tenus? Pas le moins du monde. Comment expliquer alors ce phénomène? Pour le comprendre, il faut se pencher brièvement sur les différents systèmes d'organisation de l'Etat en vigueur dans les cantons. Dans plusieurs d'entre eux en effet, notamment les cantons catholiques, les communes politiques traditionnelles sont doublées d'une commune ecclésiastique. Celle-ci tire généralement une bonne part de ses revenus des impôts prélevés sur tous les membres de la communauté. Ces communes ecclésiastiques affectent fréquemment des fonds importants à des projets dans les pays en développement; le plus souvent ces projets n'ont plus guère de connotation religieuse. Malgré le statut de droit public dont bénéficient ces collectivités, leurs contributions ne sont pas considérées comme partie intégrante de l'aide communale et cantonale au développement mais elles sont comprises dans le total de l'aide privée. Ce serait d'ailleurs pur formalisme que de le relever, si ce phénomène n'expliquait pas la singulière faiblesse des contributions de communes catholiques que l'on pourrait constater en jetant un coup d'œil superficiel sur les statistiques.

Les communautés catholiques semblent considérer fréquemment que l'aide au développement est de la compétence de la commune ecclésiastique plutôt que de la commune politique. A l'instar des exemples lucernois et saint-gallois que nous avons cités ci-dessus, une quantité de fonds publics transitent ainsi par la commune ecclésiastique pour être confiés ensuite à des organisations confessionnelles. II y aurait là 
sans doute sujet à méditer, dans la mesure où cette attitude est vraisemblament liée à une conception de l'aide, voire même du développement, assez différente de celle que l'on peut voir dans d'autres communes ou au sein de l'administration fédérale chargée de la coopération.

L'image de l'aide, telle qu'elle est perçue par les communes et les cantons donateurs eux-mêmes, ne recoupe donc pas exactement le tableau présenté par les statistiques officielles dressées chaque année par Swissaid et la Confédération. Car la statistique ne tient compte que des prestations conformes aux normes citées ci-dessus (p. 156). Elle ne peut pas être interprétée comme un classement des communes solidaires.

L'aide fournie par les communes est d'ailleurs avant tout le fait des grandes communes de Suisse. Vingt-six des trente-trois villes de Suisse qui comptent plus de 20.000 habitants participent au financement de programmes de coopération ou d'aide humanitaire. Le total de leurs versements se montait à 3.107 .000 francs en 1982, soit 79,2\% de l'aide communale comptabilisée cette année-là.

Avant d'aller plus avant à la découverte de la diversité et de la complexité des liens qui se sont créés au fil des ans entre nos communautés publiques et certaines régions en développement, il convient encore de rappeler une évidence: les communes helvétiques qui œuvrent aujourd'hui dans la coopération au développement ou l'aide humanitaire ne sont qu'une faible minorité. Relever que près de cent trente communes ont travaillé dans ce sens en 1982, c'est constater du même coup que plusieurs milliers d'autres, moins peuplées, $n$ 'ont rien fait de sensible dans ce domaine.

Du côté des cantons et demi-cantons la situation est quelque peu différente puisque vingt et un d'entre eux ont consacré une part de leur budget à l'aide au développement durant la même année. Les "cancres" de la solidarité étaient alors Appenzell (Rhodes extérieures et intérieures), Tessin, Valais et Schaffhouse, quoique ce dernier cas soit assez particulier, comme nous le verrons plus loin.

\section{HISTORIQUE}

\section{Développement de l'aide communale et cantonale en Suisse}

Le manque de statistiques précises centralisées au niveau fédéral ne permet pas de situer exactement les origines de l'aide communale et cantonale au développement dans notre pays.

II est pourtant évident que cette forme d'action repose sur de solides traditions de solidarité. Jusqu'à la fin de la deuxième guerre mondiale, les bénéficiaires de cette solidarité se trouvaient principalement en 
Suisse même ou dans des régions géographiquement proches. Le soutien financier à des causes humanitaires de la part de communes ou de cantons date parfois d'avant la guerre: la Croix Rouge a ainsi derrière elle une longue expérience de collectes auprès des collectivités publiques.

II faut attendre la fin des années cinquante pour voir les premiers pas des villes et de certains cantons suisses dans le domaine tout neuf de la coopération technique. C'est à cette époque en effet que se constituent bon nombre d'organisations privées dont le but est de favoriser le développement économique et social dans les pays en développement. Helvetas est né en 1955, Swissaid (sous le nom d'Aide suisse à l'étranger) en 1956, Swisscontact en 1959. C'est en 1961 qu'est créé à Berne le poste de Délégué à la coopération technique. La création de ces nouvelles infrastructures représente une étape décisive de I'histoire de l'aide communale et cantonale au développement; depuis cet instant, les petites collectivités disposent en effet d'instruments rationnels et fiables qui leur permettent de dépasser le niveau de l'aide en cas de catastrophe pour envisager des programmes de coopération à plus long terme.

Après quelques années de maturation, on assiste à l'aube des années septante à un véritable boom des naissances de la coopération communale et cantonale. II y a plusieurs raisons à cela. En premier lieu cette période est l'âge d'or des finances publiques. La croissance économique bat encore son plein et l'on peut songer sans trop d'inquiétude à utiliser l'argent public pour des causes qui n'étaient pas jusqu'alors du ressort des communes et des cantons. D'autre part, l'écart croissant entre les pays du Sud et du Nord démontrait clairement que les efforts entrepris jusqu'alors (et notamment par la Confédération) n'étaient pas suffisants et qu'il y avait largement place pour d'autres efforts à une échelle plus réduite. Enfin, la réflexion sur les rapports entre la Suisse et le Tiers Monde débouchait à cette époque sur toute une série de mouvements (Déclaration de Berne, Magasins du Monde, etc...) qui allaient souvent impulser ici ou là de nouveaux projets de coopération communale ou cantonale.

Le débat profond qui animait les Eglises à ce propos (4) et la Conférence interconfessionnelle Suisse - Tiers Monde de 1970 eurent aussi un impact non négligeable sur I'attitude des communes à l'égard de ce problème.

En 1968 la paroisse réformée de Münsingen organisa un séminaire sur le Tiers Monde. Les participants conclurent à la nécessité d'agir rapidement, par exemple sous la forme d'un financement de projets de coopération. Une initiative communale fut lancée pour exiger de la commune politique qu'elle consacre chaque année une part de ses recettes à cette nouvelle tâche. Malgré I'opposition déclarée de l'exécutif, I'assemblée communale accepta la proposition.

La formation en 1971 d'une Communauté de travail qui regroupait les quatre 
principales CEuvres d'entraide suisses (Swissaid, Action de Carême, Pain pour le Prochain, Helvetas) suscita aussi plusieurs tentatives visant à soutenir ses efforts. Ainsi dans le canton de Vaud où une motion de Morsier fut déposée au Grand Conseil (législatif) en novembre 1971 qui demandait à l'Etat de participer durablement à l'aide aux pays en voie de développement en remettant chaque année $1 \%$ de ses recettes fiscales à la Communauté de travail et éventuellement à d'autres associations similaires.

En octobre 1971, une motion fut déposée sur le bureau du président du Grand Conseil de Schaffhouse pour prier le gouvernement cantonal d'examiner dans quelle mesure et sous quelle forme le canton pourrait $s^{\prime}$ associer aux efforts faits en faveur des pays en développement. Cette motion était contresignée par des membres de toutes les fractions parlementaires représentées au Parlement.

Ce $n$ 'est que quelques mois plus tard que le peuple zurichois approuvait en votation populaire le principe d'une aide annuelle aux régions les plus défavorisées de Suisse et du Tiers Monde.

De nombreux exemples viennent encore démontrer que les années 1969-1973 furent une période faste pour les débuts de l'aide communale et cantonale.

Dès 1974 par contre le mouvement allait fortement s'atténuer. Le choc pétrolier de 1973 provoqua un séisme dans les milieux attachés à la politique de développement, y compris au niveau des cantons et des communes. On prit conscience un peu partout que les pays du Tiers Monde n'étaient pas forcément de frêles agneaux vivant de notre charité mais que leurs intérêts pouvaient parfois diverger. Le réveil était douloureux pour certains hommes politiques de notre pays qui s'apercevaient que les clichés misérabilistes qu'ils entretenaient auprès de leurs concitoyens et qu'ils avaient cru fondés étaient démentis par les faits. Irrités par I'audace des pays membres de l'OPEP, quelques politiciens eurent tôt fait de faire de l'aide au développement un bouc émissaire tout trouvé. L'argument était simple: "Nous n'allons tout de même pas aider des pays qui menacent notre prospérité dès qu'ils ont atteint un certain seuil de développement." Aussi grossière qu'elle soit, l'idée n'allait pas tarder à faire son chemin jusqu'aux assemblées cantonales ou communales qui délibéraient alors dans un climat fortement empreint de xénophobie. Dans la foulée des initiatives de l'Action nationale (la première est repoussée en 1970), les mouvements nationalistes et xénophobes font leur apparition dans les législatifs communaux et cantonaux.

Le 11 décembre 1974, le Dr H. Ramseier et huit de ses collègues interpellaient le Conseil de Ville (exécutif) de Zurich en ces termes : "Au cours des derniers mois, l'opinion de la population suisse a considérablement évolué à propos du problème de l'aide au développement. Les actions politiques des pays producteurs de pétrole et la majorité massive que constituent certains groupes 
d'Etats avec les pays en développement à l'ONU et dans d'autres organisations internationales ont attiré l'attention de nombreux individus dans les démocraties occidentales. C'est pourquoi nous demandons au Conseil de Ville quels sont les principes sur lesquels il s'appuie pour distribuer aux pays en développement l'aide financière mise en son temps à disposition lors d'une votation" (trad.).

Au Palais fédéral, la nouvelle Loi fédérale sur la coopération au développement et l'aide humanitaire venait d'être mise sous toit. Elle serait certainement entrée en vigueur en 1974-1975, si le Parlement fédéral, craignant un référendum inspiré par les milieux opposés à une plus grande ouverture de la Suisse sur le monde, n'avait pas préféré renvoyer la loi au dernier moment au gouvernement afin qu'il y apporte quelques retouches. Comme à Zurich, I'exécutif ajouta quelques principes légaux garantissant aux citoyens que leurs impôts ne finiraient pas dans des Etats qui n'en auraient pas réellement besoin. Et la loi passa sans encombre devant les deux Chambres en 1976.

Le canton de Thurgovie est un autre exemple du chassé croisé que les autorités effectuaient pendant cette période. Le Grand Conseil thurgovien avait accepté une proposition tendant à légiférer au niveau cantonal sur l'aide au développement. Le gouvernement thurgovien attendit tout d'abord que la loi fédérale définisse exactement le rôle des cantons en la matière. Puis, considé rant les difficultés auxquelles les autorités fédérales et les cantons voisins avaient affaire, il préféra remiser discrètement le projet dans un tiroir en attendant des jours meilleurs.

Cette période illustre à merveille l'un des obstacles essentiels d'une croissance ou d'une extension de l'aide communale et cantonale au développement. A quelque niveau que ce soit (fédéral, cantonal ou communal), les responsables craignent toujours de se voir désavouer. L'aide au développement étant un cas un peu particulier dans la mesure où les autorités craignent davantage un désaveu populaire qu'une opposition du législatif. Déjà fortes au niveau national ces appréhensions se démultiplient chaque fois que l'on descend d'un échelon dans l'organisation de l'Etat. Sont-elles justifiées? II est extrêmement difficile de le dire, du moment que très peu de votations ont eu lieu à ce sujet. Les quelques exemples sont parfois favorables à l'aide (Münsingen 1969, Zurich 1972), parfois défavorables (Genève 1982).

Les opposants à toute solidarité de la Suisse avec les pays en développement, qu'elle soit communale ou nationale, arguent régulièrement d'un fossé qui séparerait sur ce point la classe politique, toutes tendances confondues, et l'opinion publique. Il est impossible de statuer sur le bien-fondé d'une telle assertion, aucun exemple (la votation populaire de 1976 sur I'IDA) ou contre exemple (le grand succès de la pétition lancée en 1983 par les CEuvres d'entraide pour défendre l'aide fédérale au développement) n'étant véritablement probant. 
Par contre, il est intéressant de constater que les partisans de l'aide communale et cantonale tentent souvent de répondre à l'avance à ce genre de critiques. Des divers moyens utilisés pour convaincre l'opinion de la nécessité d'une aide, nous retiendrons ici les trois plus courants.

Le premier consiste à centrer les efforts de la collectivité sur l'aide humanitaire. Les secours à court terme apportés lors de catastrophes naturelles, de guerres, etc. rencontrent toujours une très grande sympathie auprès de la population. Pour les autorités, c'est donc un "produit" facilement "vendable". Certaines communes et parfois même certains cantons (Bâle-Campagne, Grisons, Thurgovie, Zoug, Nidwald) préfèrent se contenter de l'aide humanitaire et $n^{\prime}$ accordent aucun crédit pour la coopération au développement (à plus long terme). Certains partisans de la coopération n'hésitent d'ailleurs pas à accuser les responsables politiques de ces cantons de se défiler face à leurs responsabilités et d'éviter ainsi un débat pourtant nécessaire dans I'opinion publique. L'attitude de ces quelques cantons est de moins en moins fréquente. La majorité des cantons et bon nombre de communes favorisent à la fois les deux composantes de l'aide au développement.

Le deuxième revient à donner un maximum de garanties à la population locale sur la manière dont l'aide est gérée et sur ses destinataires. Certaines communes ont même fixé par écrit un certain nombre de principes auxquels les prestations sont soumises. (Voir ci-dessous, pp. 174 ss.) Généralement ces communes désignent comme seuls bénéficiaires possibles les couches les plus pauvres des pays en développement. C'est d'ailleurs aussi ce qu'avait fait le Conseil fédéral à l'article 5 de la Loi fédérale sur la coopération au développement.

Enfin, l'un des grands atouts de la coopération à petite échelle est de pouvoir chercher un ancrage local aux liens avec un pays du Tiers Monde.

Lorsque, en 1981, les autorités yverdonnoises eurent pour la première fois à choisir un projet de développement, elles avaient sous les yeux cinq à six dossiers possibles. Elles décidèrent finalement de soutenir un projet en Tunisie parce que ce pays était fréquenté chaque année par de jeunes Vaudois (dont des Yverdonnois) qui allaient travailler dans la région. Cet accrochage bien concret eut raison d'autres arguments inspirés par des principes de politique de développement (le pays bénéficiaire est-il un bénéficiaire prioritaire?, etc.).

Après les années difficiles 1973-1977, I'aide communale et cantonale au développement a repris son rythme de croisière. Le nombre de communes portant des crédits d'aide à leur budget reste sensiblement identique au fil des ans. Le montant total de l'aide au développement communale a sensiblement baissé de 1979 (3,9 millions) à 1981 (3,3 millions) pour augmenter à nouveau en 1982 (env. 4 millions). II faut 
d'ailleurs relever que cette évolution s'explique avant tout par la chute libre du montant consacré à l'aide humanitaire qui a passé de 1,6 millions en 1979 à 540.000 francs en 1982. La coopération au développement progresse par contre très régulièrement.

Une évolution parfaitement identique peut être observée dans le cadre de l'aide cantonale qui a passé de 3,5 millions en 1979 à 2,7 millions en 1981 pour remonter à 3,2 millions en 1982; tandis que la composante d'aide humanitaire chutait de 1,8 millions en 1979 à 732.000 francs en 1982.

\section{Comment démarre l'aide au développement?}

Caisse de résonance des humeurs de la population, c'est généralement le parlement local qui est l'artisan des projets d'aide communale et cantonale au développement. C'est le cas en tout cas lorsque les projections financières dépassent les compétences de l'exécutif. Après quelques semaines ou quelques mois de tractations plus ou moins discrètes à l'intérieur des partis, le projet finit par prendre corps sous forme de motion.

A Yverdon, lors de la séance du Conseil communal du 28 juin 1979, le conseiller $F$. Deluz (PDC) monte à la tribune pour demander à ses collègues d'ouvrir de nouvelles relations avec le Tiers Monde. II propose notamment:

"- de prévoir chaque année au budget une somme, pourcentage des dépenses, destinée à une réalisation dans le Tiers Monde;

- que la réalisation touche une petite communauté, un village;

- qu'une commission propose chaque année au Conseil communal un ou plusieurs projets préparés par des organismes connus».

Après I'approbation par la Municipalité, le Conseil accepta la motion à l'unanimité moins trois voix et la renvoie à la Municipalité pour étude et rapport.

Au Grand Conseil vaudois, c'est une députée libérale, Mme Odile de Morsier, qui intervient en novembre 1971 pour proposer que $1 \%$ du produit des impôts cantonaux soit affecté au financement de projets d'aide aux pays en voie de développement. La somme serait remise à la Communauté de travail Swissaid, Action de Carême, Pain pour le Prochain, Helvetas qui utiliserait les fonds après décision du Conseil d'Etat. Sans débat, le Grand Conseil renvoie la motion au Conseil d'Etat pour étude et rapport. La réponse ne viendra que dix ans plus tard, et elle sera négative: le gouvernement cantonal refuse les conclusions de la motion de Morsier tout en assurant que l'œuvre de coopération technique en cours (le canton de Vaud finance un programme de formation professionnelle en Tunisie depuis 1972) sera poursuivie; mais le financement continuera à être assuré par des montants variables mis chaque année au budget ordinaire.

En 1969 le socialiste thurgovien A. Engeli dépose lui aussi une motion chargeant le gouvernement local de mettre sur pied une nouvelle loi cantonale qui régirait l'aide fournie par la Thurgovie dans le domaine de l'aide humanitaire comme de la coopération au développement. Surprise, la motion est adoptée par le Conseil par 56 voix contre 55 . Un projet de loi est alors élaboré par le 
gouvernement. Ce projet sera remanié de fond en comble par la Commission préparatoire dirigée par le Dr Onken (PS). En 1977, I'entrée en matière sur le projet définitif est acceptée au Grand Conseil par 62 voix contre 57 . Mais dans la minute qui suit, le député $H$. Bommer (PDC) propose de limiter le champ de la loi à l'aide humanitaire et d'amputer le projet de toute la partie traitant de la coopération au développement, en fait I'essentiel du projet. La proposition Bommer est adoptée par 56 voix contre 55 . Au désespoir des membres de la commission, huit ans d'efforts sont réduits à néant.

Les trois cas ci-dessus sont naturellement choisis par hasard, et il serait risqué de vouloir en tirer quelque règle générale que ce soit. Cependant dans ces exemples comme dans d'autres, on remarquera la diversité des promoteurs de l'aide au développement. De l'extrêmegauche à la droite libérale, les partis politiques suisses comptent tous des partisans d'une plus grande coopération des collectivités suisses avec le Tiers Monde.

Ceci dit, le jugement mérite d'être nuancé. Car si aucun parti ne peut prétendre avoir le monopole en ce domaine, d'autres sont régulièrement réticents. Le parti radical est ainsi, dans la quasi totalité des cas rencontrés lors de cette petite enquête, la force politique locale qui a exprimé le plus de réserves à l'encontre des innovations en matière d'aide au développement. Pour ne pas parler des Républicains ou de l'Action nationale qui y sont carrément hostiles.

Outre les partis, d'autres forces sont actives sur le terrain politique local, lorsqu'il s'agit de pousser les autorités à agir. L'un des acteurs essentiels est fréquemment l'Eglise locale, qu'elle soit catholique ou protestante.

Dans la commune bernoise de Münsingen, les Eglises ne se contentèrent pas de lancer une initiative communale pour financer un projet de développement (cf. ci-dessus p. 101) mais elles rassemblèrent elles-mêmes année après année des fonds pour les joindre à ceux que la commune politique amenait. En 1982 encore, alors que les frais du projet péruvien pris en charge se montaient à environ 120.000 francs, la paroisse de Münsingen en assumait la moitié.

Les représentants de la communauté religieuse siègent d'ailleurs dans le comité qui gère le projet. L'Eglise catholique, moins implantée à cet endroit, contribue elle aussi au financement de l'aide.

Les organisations militantes spécialisées en politique de développement sont parfois à l'origine des efforts d'une commune.

A Yverdon, le motionnaire qui permit à l'aide communale de démarrer était un membre actif des Magasins du Monde, une organisation formée de bénévoles qui tentent $d^{\prime}$ instaurer un commerce plus juste entre la Suisse et les pays en développement. 
Enfin, des coïncidences, des conditions tout à fait uniques peuvent quelquefois expliquer les décisions d'une commune. Ici un membre de l'exécutif connaît un coopérant ou un missionnaire qui travaille dans un bidonville du Brésil ou d'ailleurs. Là, un membre du gouvernement cantonal entretient des relations d'amitié avec un dirigeant d'un pays du Tiers Monde. Un déclic suffit pour engager toute la collectivité dans un effort de solidarité.

Le canton du Jura représente un cas tout à fait unique. Lors de sa création, l'Assemblée constituante introduisit aussitôt un article faisant de la coopération une tâche de l'Etat prévue par la Constitution.

Les Jurassiens expliquent cette disposition par le climat politique particulier qui prévalait lors de la fondation du canton du Jura. Après avoir bénéficié pendant les années de lutte de la solidarité agissante de nombreuses communautés extérieures au Jura, le nouveau canton désirait en quelque sorte leur rendre la politesse.

La solidarité jurassienne s'exerce en cercles concentriques puisqu'elle vise d'abord les voisins du nouveau canton, puis les communautés francophones à travers le monde et enfin les pays en développement.

\section{COLLECTIVITES LOCALES ET CONFEDERATION}

\section{L'aide au développement: domaine réservé de la Confédération?}

L'article 8 de la Constitution fédérale donne à la Confédération la compétence de conclure des traités avec l'étranger et fait plus largement de la politique extérieure l'une des activités de l'Etat central.

L'aide au développement et les rapports avec les pays du Tiers Monde partie intégrante de la politique extérieure suisse devrait-elle alors être de l'unique ressort de la Confédération?

C'est ce qu'affirment de nombreux détracteurs de l'aide cantonale ou communale. L'argument a ainsi été utilisé lors de la campagne contre I'initiative genevoise dite "initiative 0,7 " qui proposait que le canton de Genève affecte chaque année à la coopération au développement un montant équivalant à $0,7 \%$ de son produit cantonal brut. "Laissons cela à la Confédération qui est mieux outillée et qui dispose de plus de moyens" disaient en substance les opposants à l'initiative.

En réalité, la Confédération laisse une large place aux cantons et aux communes qui désirent être actifs dans ce domaine. L'article 12 de la Loi fédérale sur la coopération au développement et l'aide humanitaire précise : "Le Conseil fédéral peut collaborer avec des cantons, des communes et des institutions publiques à des activités qui relèvent de la coopération au développement et de l'aide humanitaire internationales et soutenir leurs initiatives." 
Dans le message qui accompagnait la loi lors de son examen par les Chambres, le Conseil fédéral était encore plus explicite : «...un nombre croissant de cantons et de communes ont, au cours des dernières années, apporté leur contribution à la coopération au développement et à l'aide humanitaire. Nous nous en félicitons. En Suisse, c'est généralement de bas en haut que se forme l'opinion publique et que s'anime la vie politique; $c^{\prime}$ est donc dans les cantons et les communes que l'on porte tout d'abord attention aux tâches nouvelles de la collectivité, avant qu'elles ne soient reprises dans la politique fédérale. Par contre, à l'image d'ailleurs de la plupart des tâches de politique étrangère, la coopération au développement et l'aide humanitaire internationales, dont les résultats dans de lointains pays ne sont pas visibles à notre population, ont été par la force des choses abordés au niveau de la politique fédérale sans avoir suivi la voie que nous venons de décrire. La participation des cantons et des communes à la coopération au développement et à I'aide humanitaire internationales en favorisera l'enracinement dans l'opinion publique» (5).

Auparavant, lors de la procédure de consultation sur le projet de loi, les cantons avaient déjà annoncé leur profond intérêt pour la loi en général et pour l'article 12 en particulier. Sans discuter le principe de la compétence fédérale en la matière, le canton de Thurgovie annonçait d'emblée son intérêt pour la place que le projet de loi laissait aux communes et aux cantons. Zoug donnait aussi son accord à cette formule, de même que les cantons d'Argovie et de Saint-Gall qui souhaitaient que les droits que l'on accordait aux petites collectivités n'aient pas de conséquences contraignantes. "Les cantons ne devraient pas être obligés d'œuvrer dans le secteur de la coopération", annonçaient prudemment plusieurs cantons. Le Conseil fédéral les rassura sur ce point dans son message: "L'article 12, toutefois, ne fait que mentionner la possibilité d'une participation et n'a pas pour but de reporter les tâches qui incombent à la Confédération sur les cantons et les communes, ou encore de les obliger à assurer une contribution à la coopération au développement et à l'aide humanitaire internationales" (6).

La question est réglée, mais elle $n$ 'en suscite pas moins quelques controverses à l'occasion. Plus d'un siècle d'existence de l'Etat fédéral ne suffit pas à effacer tous les sujets de discorde possible quant au partage des tâches Confédération-cantons. Et cela d'autant plus que I'aide au développement est une activité nouvelle que les rédacteurs de notre Constitution ne pouvaient pas prévoir en 1874.

Le canton du Jura, enfant terrible de la Confédération, a posé plusieurs fois d'épineux problèmes aux juristes de la Couronne lorsqu'il s'est agi de ses relations avec l'extérieur. En 1978, une délégation gouvernementale seychelloise arrivait à Delémont, conduite par un ministre venu rendre visite à des missionnaires jurassiens qui avaient longtemps enseigné aux Seychelles. Une rencontre officielle fut mise sur pied avec les autorités du nouveau canton. 
L'entente fut si totale que les deux parties convinrent d'un accord de coopération. La Confédération qui n'était pas partie à l'accord fut piquée au vif et le fit savoir au gouvernement jurassien en lui rappelant que la conclusion de traités est du ressort exclusif de l'Etat fédéral.

En juillet 1983, la question a d'ailleurs resurgi à propos de la signature d'un traité de coopération culturelle entre le Jura et le Québec. Un tel traité doit-il être paraphé par la Confédération? Berne et Delémont n'ont pas réussi à se mettre d'accord (7).

$D^{\prime}$ autres problèmes restent ouverts. Certains fonctionnaires cantonaux chuchotent qu'ils souhaiteraient une nouvelle répartition des tâches entre Confédération et cantons. Berne garderait la coopération au développement tandis que les autres collectivités publiques se chargeraient de l'aide humanitaire.

Cette opinion a été notamment exprimée au cours des débats du Grand Conseil thurgovien à propos du projet de loi cantonale sur l'aide au développement. Prenant la parole au nom du groupe démocrate-chrétien (le parti le plus important du canton), Franz Bommer déclarait: "L'aide internationale au développement (entendue ici au sens de coopération au développement, $\mathrm{Ndr}$ ) est I'affaire de la Confédération. II en va autrement pour l'aide humanitaire... Le Conseil d'Etat doit présenter un nouveau projet en se limitant à l'aide humanitaire" (trad.).

Le Parlement fut du même avis et élimina du projet toute référence à la coopération à plus long terme.

Les arguments des partisans de cette nouvelle répartition des tâches sont d'ordre technique et politique. Technique: I'aide humanitaire serait une formule de solidarité plus facile à gérer; elle serait moins facilement gaspillée et le risque de voir les montants récoltés finir dans les poches d'intermédiaires corrompus serait moindre lui aussi. L'argumentation politique est plus convaincante: la population locale, frappée par l'aspect spectaculaire des catastrophes, n'a pas besoin d'être convaincue du bien-fondé de l'aide. Les collectivités locales pourraient ainsi amasser plus de fonds pour cet objectif tout en permettant à la Confédération de consacrer tous ses efforts à la coopération technique et financière.

Une telle solution n'est pas pour plaire à la Confédération qui compte justement sur les cantons et les communes pour se faire l'avocat de la coopération à plus long terme auprès de la population. La tâche est ingrate, c'est certain. Raison de plus pour ne pas la laisser entièrement à la Confédération qui a déjà assez à faire pour défendre ses propres projets, attaqués plus souvent qu'à leur tour par quelques politiciens en mal de publicité.

Par ailleurs on imagine assez mal la Suisse refuser toute aide humanitaire au moment d'un cataclysme en expliquant que c'est là la tâche 
des communes et des cantons. Une telle attitude ne manquerait pas d'avoir des répercussions fâcheuses sur notre politique extérieure.

La formule a donc bien peu de chance d'aboutir.

\section{L'apport de la Direction de la Coopération au développement et de l'Aide humanitaire (DDA)}

Les autorités fédérales, et plus particulièrement la Direction de la Cooopération au développement et de l'Aide humanitaire (DDA) sont souvent appelées à assister les communes ou les cantons lorsque ceux-ci s'initient aux activités de I'aide au développement.

La DDA peut déjà intervenir au moment du choix d'un projet. Lorsqu'une commune s'adresse à elle afin de savoir où son argent serait le plus utile, l'administration fédérale renvoie généralement la commune à l'une des œuvres d'entraide privées. Ces dernières tiennent en effet à disposition des collectivités qui en font la demande une sorte de "catalogue» de projets dans divers secteurs et sur différents continents. La commune a ainsi la possibilté de faire un choix en fonction de ses propres critères.

Dans de très rares cas la DDA conseille directement la commune en lui proposant un projet.

Lorsqu'en 1980 la commune d'Yverdon procéda à un appel d'offres de projets auprès de différentes œuvres sociales, elle s'adressa aussi à la DDA qui lui proposa de se joindre aux communes des environs de Nyon qui soutenaient déjà un projet en Tunisie. Cette idée agréa aux autorités yverdonnoises, soucieuses de ne pas laisser les efforts se disperser.

De nombreux autres services sont rendus aux cantons et aux communes par la DDA.

La commission du Grand Conseil thurgovien chargée de présenter à l'assemblée plénière un projet de loi cantonale régissant l'aide au développement s'assura aussi la collaboration d'un représentant de l'administration fédérale qui renseigna les membres de la commission sur ce qui s'était fait ailleurs en Suisse en la matière.

L'administration cantonale jurassienne fait aussi fréquemment appel aux compétences techniques de la DDA dans le cadre de la coopération cantonale avec les Seychelles.

La Ville de Zurich a constitué une commission consultative municipale qui a pour fonction de sélectionner les projets qui seront soutenus financièrement par la Ville et de conseiller l'exécutif qui prend les décisions en dernier ressort. Dans cette commission un représentant de la DDA siège aux côtés de délégués des CEuvres d'entraide, du législatif et de l'administration municipale.

Le canton de Zurich procède lui aussi par appel d'offres de projets. Chaque année il doit donc faire un tri. Pour s'assurer de la qualité de son choix, il soumet ensuite les projets sélectionnés à l'administration fédérale qui lui donne ou non son feu vert après examen approfondi. 
Münsingen, seule commune suisse à financer entièrement et directement un projet de développement à l'étranger, bénéficie aussi du soutien actif de la Confédération qui transfère les fonds remis par la commune jusqu'au Pérou et qui en contrôle ensuite l'utilisation sur place.

On le voit, la Confédération est loin d'être inactive lorsqu'il s'agit d'assister les communes et les cantons dans leurs efforts. Dès 1969, la DDA confia à un fonctionnaire la tâche de coordonner les initiatives locales liées à l'aide au développement. L'article 12 de la loi fédérale vint d'ailleurs lui confirmer ce mandat quelques années plus tard. Malheureusement, du fait du blocage du personnel fédéral imposé par les Chambres (blocage particulièrement gênant pour la DDA, frappée en pleine croissance), ce poste dut être abandonné. La coordination de I'aide communale et cantonale est désormais fractionnée entre plusieurs fonctionnaires.

\section{LE CONTENU DE L'AIDE}

\section{Généralités}

A nouveau, il est extrêment difficile de définir le prototype du projet de développement qu'une commune ou qu'un canton a choisi de financer. Pour ce faire il serait nécessaire de posséder des données statistiques beaucoup plus fournies que celles dont nous disposons aujourd'hui.

Néanmoins, le témoignage des responsables des CEuvres d'entraide qui sont mandatés par la quasi totalité des communes et des cantons donateurs pour gérer et contrôler l'utilisation de l'aide sur le terrain permet de cerner quelques-uns des grands traits du contenu de l'aide communale et cantonale au développement. Lorsqu'une collectivité publique s'adresse à l'une ou l'autre des organisations privées de développement, celle-ci lui propose toujours un éventail de projets non encore totalement financés. De manière à laisser le plus grand choix possible aux autorités locales, les CEuvres d'entraide veillent à présenter des projets situés dans divers continents, et touchant des secteurs économiques différents: développement rural, irrigation, artisanat, éducation, santé, travail social, etc... Les organisations liées à l'Eglise présentent au surplus des projets liés à une activité missionnaire (construction d'église, fourniture de matériel, entretien de personnel ecclésiastique expatrié, etc...) dont le financement n'est pas comptabilisé dans I'aide publique.

Quels sont les pays privilégiés par les contributions communales et cantonales? Dans la plupart des cas, les projets financés se situent dans les Etats les plus pauvres de la planète. En cela, les collectivités locales ne font qu'imiter l'aide apportée par la Confédération. Mais si 
cette dernière est contrainte par la loi (art. 5 de la Loi fédérale de la coopération au développement) d'accorder la priorité aux habitants les plus démunis du globe, la règle est nettement moins stricte pour les communes qui n'ont généralement pas de textes légaux à ce propos là quelques intéressantes exceptions près que nous examinerons plus loin). Le niveau de développement économique d'un pays est un critère parmi d'autres, lorsque des autorités locales ont à trancher entre plusieurs propositions de projets. Et il est intéressant de constater que l'intérêt porté à ce critère décroît lorsque l'instance décisionnelle n'est par formée ou conseillée par des spécialistes de la coopération.

Tous les projets financés par la Ville de Zurich au cours des dix dernières années ont pour cadre les pays les plus pauvres du Tiers Monde là une exception). Mais Zurich est aussi une des rares communes à bénéficier de l'appui de spécialistes de la DDA et des œuvres d'entraide. Par ailleurs, le Conseil de Ville a aussi posé un certain nombre de principes écrits qui doivent prévaloir lors de la sélection de projets. La priorité aux pays les plus démunis est l'un de ces principes.

Le canton de Vaud concentre la majeure partie de son aide en Tunisie. Ce pays ne figure pas parmi les Etats les plus défavorisés de la planète. Le responsable cantonal reconnaît lui-même que des choix plus judicieux pourront être faits maintenant que le projet de coopération avec la Tunisie touche à sa fin. En 1972 , lors des débuts de la coopération vaudoise au développement, le choix d'un secteur d'activité (la formation professionnelle) avait joué un rôle important dans la décision; par ailleurs, la Tunisie était alors un partenaire très prisé par les coopérations au développement publiques et privées

Plus que le niveau de développement économique atteint par le pays, c'est souvent la nature du régime politique en place qui préoccupe les communes ou les cantons.

Lors de la procédure de consultation sur le projet de Loi fédérale sur la Coopération au développement et l'Aide humanitaire, quelques cantons avaient déjà attiré l'attention du législateur fédéral sur ce point. Le canton du Tessin souhaitait ne voir l'aide accordée qu'à la condition que les droits de I'homme soient respectés. Le canton d'Uri voulait voir exclure de la liste des bénéficiaires les pays qui protègent le terrorisme ou qui violent les principes de la communauté internationale.

Les principes d'application de l'aide municipale zurichoise sont très clairs sur ce point : "Dans le cadre de l'aide au développement, il ne peut pas être tenu compte du système de gouvernement en place dans le pays en développement. Si l'on excluait de la liste des pays soutenus tous les pays en développement dirigés de manière autoritaire, il ne subsisterait que peu d'Etats qui pourraient être pris en considération. L'aide au développement de la Ville est destinée aux habitants d'un pays et non à son gouvernement. En général, les énormes masses qui vivent dans la pauvreté $n$ 'ont pas d'influence sur le pouvoir. 
Dans certains cas d'exception l'attitude d'un Etat sur la scène internationale ou sa situation politique interne doivent pourtant être prises en considération. L'aide au développement en tant qu'acte de la solidarité internationale n'est possible que lorsqu'elle est portée par l'opinion publique du pays donateur. Un pays en développement dont le comportement est contraire aux intérêts de la paix, qui viole les droits de l'homme les plus élémentaires ou qui s'attire des reproches justifiés en Suisse, ne peut naturellement pas être soutenu. On ne peut apprécier de tels comportements que dans des cas particuliers" (8) (trad.).

Si l'on consulte la liste des pays qui ont bénéficié de financement de la part de la Ville de Zurich, on constate qu'aucun Etat dirigé par un régime progressiste n'y figure. Les responsables confirment que la Ville a renoncé à aider des pays comme le Nicaragua ou l'Ethiopie, tandis que le Zaire par exemple continue à obtenir des crédits. La Ville évite pourtant de soutenir des projets dans des pays dirigés par des dictatures d'extrême droite, tels que le Guatemala ou le Honduras. L'attitude des autorités municipales en la matière est dictée par le climat politique (Zurich est dirigée par une majorité politique de centre droite) et par l'objectif d'éviter toute polémique dans l'opinion publique.

Le canton de Zurich mène une politique parfaitement identique à cet égard. Echaudées par un article de presse qui leur reprochait un peu hâtivement de n'aider le Nicaragua que lorsque des dictateurs étaient en place, les autorités cantonales ont pris le parti d'éviter à tout prix qu'un tel cas se représente. L'aide n'est donc accordée qu'à des pays "dont on ne parle pas». Cette attitude timide est aussi sujette à caution si l'on pense que les pays en crise économique et sociale perpétuelle sont évidemment ceux dont la vie politique est la plus troublée par des coups d'Etat ou par des conflits internes.

En ce qui concerne l'aide humanitaire, destinée à panser rapidement les plaies provoquées par une catastrophe, les critères géographiques et politiques ne sont plus déterminants: chacun s'efforce alors d'aider au mieux et le plus vite possible pour soulager la détresse des populations concernées. Parfois pourtant, des efforts supplémentaires sont dus à une motivation politique implicite.

La Chaux-de-Fonds, commune à majorité politique de gauche, a répondu plusieurs fois ces dernières années aux sollicitations de parlementaires locaux qui demandaient à l'exécutif de fournir assistance à des individus ou des groupes réprimés ou frappés à cause de leurs convictions politiques (Turquie, Pologne, Palestiniens au Liban, etc...).

Quels sont les secteurs économiques privilégiés par l'aide des communes et des cantons suisses? "Difficile à dire» répondent K. Matter, secrétaire de Swissaid et $M$. Küttel, responsable du service de projets des CEuvres d'entraide catholiques. "Mais il est clair que les communes privilégient fortement les investissements permettant des réalisations visibles." Soucieuses de rendre des comptes à leurs électeurs, les autorités se tournent ainsi automatiquement vers un certain type de projets: construction de puits ou de canaux, de bâtiments, installa- 
tions destinées à l'élevage, etc... Par contre, tous les projets qui n'affectent pas des structures visibles sont défavorisés - c'est le cas notamment des projets éducatifs et du travail social. Au sein des organismes liés à l'Eglise catholique, on perçoit parallèlement une évolution dans les choix au bénéfice des projets non confessionnels. Là aussi la crainte d'indisposer une minorité de citoyens d'autres confessions est à l'origine du phénomène.

L'exemple de Zurich illustre bien cette tendance.

Dans les principes directeurs de son aide au développement, la commune de Zurich a fixé la règle suivante: «...3. L'aide au développement de la Ville de Zurich est $d$ 'abord une aide d'investissement. Des contributions ne seront accordées qu'à des réalisations concrètes...» (9).

Le choix des communes et des cantons est aussi influencé par la taille des projets. Les collectivités locales montrent beaucoup de réticence à soutenir de grands projets à l'étranger. Tout d'abord parce qu'elles ne croient le plus souvent pas à l'efficacité de ce type de projets. Mais aussi parce qu'elles préfèrent financer elles-mêmes l'ensemble ou en tout cas une part déterminante du projet. La nécessité de leur aide apparaît alors plus clairement que si leur contribution était noyée parmi les fonds de plus gros donateurs.

Ce facteur explique notamment l'intérêt que le canton du Jura a porté rapidement aux Seychelles. Les îles de l'Océan Indien et le nouveau canton comptent approximativement le même nombre d'habitants, donnant une échelle humaine à la solidarité entre les deux Etats. La formule de la concentration de l'aide sur un partenaire principal adoptée par le Jura, mais aussi par le canton de Vaud, les communes de Münsingen ou d'Yverdon, est fondée sur l'espoir que l'identification de l'aide à un seul projet de dimension forcément modeste donnera une meilleure assise de l'aide au développement au sein de la population.

Le contenu de l'aide au développement communale et cantonale est donc souvent déterminé par l'image que les autorités vont devoir défendre auprès des citoyens de l'endroit. En cela il est soumis à des contingences d'ordre quasiment émotionnelles qui n'affectent pas I'aide fédérale, ou en tout cas pas dans la même mesure. Ce problème resurgit chaque fois qu'il est question d'opter entre l'aide humanitaire et la coopération au développement. A vrai dire, ce n'est pas l'efficacité de l'aide humanitaire qui lui donne un avantage, c'est son aspect spectaculaire et émotionnel. La chose apparaît de manière particulièrement évidente dans les rares cas où la coopération technique parvient aussi à faire appel aux sentiments plutôt qu'à la raison.

La présentation en avril 1983 par la Télévision romande d'une émission "Temps Présent» évoquant la situation critique de l'hốpital de Ouagadougou 
(Haute-Volta) a suscité un écho extraordinaire auprès des spectateurs qui ont massivement répondu à l'appel financier lancé par les réalisateurs en faveur d'un projet de coopération avec l'hópital. Curieusement, ce projet a provoqué l'intérêt d'un certain nombre de communes, jusque-là inactives, telle que la petite bourgade d'Orbe (Vaud) où les autorités ont versé une petite somme en appelant la population locale à suivre leur exemple.

\section{Quelle politique de développement?}

On le voit, les motivations qui inspirent les communes ou les cantons dans leurs activités de coopération sont fort diverses. Elles sont souvent le fait des individus qui composent les différents organes de la collectivité et des contraintes locales auxquelles ils doivent se soumettre. Le risque est grand, dans ces conditions, qu'un changement du personnel politique affecte sérieusement le contenu de l'aide. Parfois au détriment de lidée première, défendue par tel parlementaire ou par tel groupe particulièrement motivé. Mais peut-être aussi au détriment des principes reconnus de politique de développement. Pour se prémunir contre ce risque certaines des collectivités locales ont défini un certain nombre de principes écrits auxquels les prestations d'aide au développement doivent ensuite souscrire.

La commune d'Yverdon a fixé d'emblée quatre principes d'action d'ailleurs identiques à ceux qui ont été adoptés par les CEuvres d'entraide:

- travailler à la base;

- aider I'homme à s'aider lui-même;

- viser un développement spécifique;

- travailler en partenaire.

La Ville de Zurich a donné un caractère encore plus élaboré à ces principes. Le principe d'une aide au développement communale ayant été adopté par les citoyens en votation populaire, le législatif a fixé un certain nombre de principes directeurs ayant rang de "loi» communale. Le département responsable de la gestion de cette aide a ensuite précisé les règles de conduite en la matière, équivalentes d'un règlement. A Zurich, ces principes sont au nombre de cinq:

1. Seuls peuvent être bénéficiaires de l'aide les Etats qui ne disposent pas de moyens propres suffisants. (De ce fait, tous les pays arabes sont par exemple exclus.)

2. L'aide doit être véhiculée par des organisations suisses actives dans le domaine de la politique de développement auxquelles des fonds publics peuvent être confiés.

3. L'aide doit être en premier lieu une aide d'investissement. Une contribution à des dépenses d'entretien ne peut se concevoir qu'à titre d'assistance de départ.

4. Les réalisations doivent être rapides.

5. Seuls peuvent être soutenus les projets dont l'exécution complète peut être assurée grâce aux prestations de la Ville de Zurich. 
Lors de sa tentative avortée de mettre sur pied une loi cantonale d'aide au développement, le canton de Thurgovie avait lui aussi défini des lignes de conduite relativement précises:

1. La coopération au développement doit viser les pays en développement les plus pauvres.

2. Et plus particulièrement encore les couches les plus défavorisées de la population de ces pays.

3. L'aide sous-tend les efforts propres des partenaires. Les mesures prévues doivent être élaborées et soutenues par la population concernée.

4. La priorité doit être accordée à l'amélioration de l'alimentation, à la promotion de l'artisanat, à la mise en place de services de santé accessibles à tous et à I'organisation d'un système de formation qui permette à la population de se prendre elle-même en charge.

L'adoption par des collectivités locales de ce type de principes de politique de développement présente surtout l'avantage de fixer un certain consensus sur le sens de l'action et d'éviter par la suite des malentendus ou des dérapages. Le législateur local a ainsi l'occasion de débattre des questions essentielles et de s'assurer un cadre de références grâce auquel il pourra évaluer le travail des organes de l'aide, que ces organes soient intégrés ou non à l'administration locale.

Dans la très grande majorité des cas pourtant, les communes et les cantons ne s'embarrassent pas de toutes ces précautions. D'autant plus que l'aide au développement n'est pour eux qu'une activité subsidiaire. Certains fonctionnaires avouent même qu'ils préfèrent disposer d'un maximum de marge de manœuvre pour adapter le mieux possible le projet aux conditions mouvantes de la réalité dans les pays en développement.

Le programme de coopération du canton du Jura sur l'archipel des Seychelles a ainsi vu son contenu se modifier radicalement. Pendant trois ans I'aide jurassienne a principalement consisté à affecter aux Seychelles des coopérants volontaires dans l'enseignement.

L'expérience démontre que ce type d'aide n'était vraisemblablement pas la plus adéquate, ni surtout la plus urgente pour un pays comme les Seychelles. Les autorités jurassiennes ont donc décidé de remodeler leur aide à partir de 1983 en la transformant en un appui technique au Ministère du Plan, au Département d'ingénierie de l'Ecole polytechnique (équivalant à un second cycle du secondaire) et aux bibliothèques publiques.

L'absence de principes renforce surtout un risque extrêmement sérieux pour toutes les communes et tous les cantons: celui de laisser I'aide au développement complètement à l'écart des discussions publiques. Si le Parlement n'a jamais l'occasion de débattre du sujet en dehors du budget, il est illusoire d'espérer que la population s'en préoccupe. Pire encore: si la gestion de l'aide est confiée à un fonctionnaire travaillant dans une tour d'ivoire, sa pratique en la matière risque finalement d'être l'unique critère de politique de développement. 
C'est une situation que nous avons rencontrée et dans laquelle, en l'absence d'information et de débats, la coopération au développement n'a évidemment aucun effet sur la collectivité donatrice qui n'est souvent même pas consciente de l'existence d'un programme d'aide.

\section{LES FORMES DE L'AIDE}

\section{L'aide humanitaire}

Par définition, I'aide humanitaire est une aide d'urgence, ponctuelle et imprévisible. Son acheminement sur les lieux de la catastrophe dépend donc de structures bien rodées qu'une collectivité locale ne pourrait en aucun cas mettre sur pied.

$C^{\prime}$ est pourquoi les communes et les cantons utilisent les compétences d'organisations spécialisées telles que la Croix Rouge Suisse, I'Entraide protestante, Caritas, etc... Les fonds sont rassemblés par les organisations humanitaires à l'occasion de leurs collectes annuelles respectives ou par des appels spéciaux lancés à l'occasion de situations d'urgence (tremblement de terre, guerre civile, etc...).

L'opération ne présente pas de difficultés particulières pour les collectivités publiques du moment qu'elles disposent soit d'un poste budgétaire prévu pour ce genre de cas, soit de réserves que l'exécutif peut utiliser à ce titre dans la limite de ses compétences financières.

Le Conseil d'Etat thurgovien peut dépenser jusqu'à 10.000 francs sans devoir en référer au Parlement cantonal. Cette petite marge lui permet de verser chaque année cette somme comme contribution d'aide humanitaire.

Lors des débats sur le projet de loi cantonale, il fut notamment question d'élever cette limite à 100.000 francs, mais cette proposition a été enterrée avec le projet de loi en 1982.

La Ville de la Chaux-de-Fonds porte chaque année à son budget la somme de 100.000 francs sous la rubrique "aide". Cette somme permet à l'exécutif d'intervenir à plusieurs reprises durant l'année pour financer des actions humanitaires. En 1982, par exemple, la Ville a dépensé ainsi 73.000 francs.

II ne serait pas impensable de voir une partie de cette somme affectée au financement de projets de coopération en lieu et place de l'aide humanitaire. La formule ne s'y prête cependant guère du moment qu'il est impossible de prévoir quels montants seront encore nécessaires avant la fin de l'année. Peut-être pourrait-on imaginer que le reliquat soit destiné à un projet plutôt que de rejoindre le compte de pertes et profits?

Consciente des handicaps que présenterait un système où un même budget servirait à financer la coopération au développement et l'aide humanitaire, la commune de Zurich a tenu à distinguer expressément les postes. Un budget d'aide au développement est voté annuellement au bénéfice de la coopération avec le Tiers Monde d'une part et avec les régions défavorisées de Suisse 
d'autre part. Le montant de cette aide est donc clairement établi avant même le début de l'exercice budgétaire.

Par contre, lorsque le besoin d'assistance humanitaire se fait sentir, l'exécutif municipal fait alors appel à des réserves financières qu'il n'entame qu'avec l'accord du législatif.

\section{La coopération au développement}

Dans le domaine de l'aide à plus long terme, les formes sont nettement plus variées. Les deux principales sont I'aide «indirecte» où la contribution communale ou cantonale passe par l'intermédiaire d'une CEuvre $d^{\prime}$ 'entraide. Et l'aide "directe» où la collectivité gère elle-même les fonds en créant un véritable service de coopération technique à son échelle.

La formule de l'aide «indirecte» est de loin la plus courante. Elle permet aux communes et aux cantons qui l'utilisent de disposer d'un maximum de garantie de compétence et d'efficacité. Les CEuvres d'entraide peuvent aussi coordonner les différents efforts de communes en cumulant par exemple les fonds versés par plusieurs d'entre elles pour financer un projet un peu plus vaste. Mais l'aide «indirecte» évite surtout aux petites collectivités de notre pays d'avoir à mettre en place une infrastructure spéciale ou d'alourdir leur administration. En ce sens les organisations privées de coopération au développement sont à même de fournir un véritable service aux communes et aux cantons qui s'adressent à elles.

Le canton et la Ville de Zurich sont tous deux partisans de cette solution. En 1982 , ils ont transféré l'ensemble de leurs fonds destinés à la coopération au développement $\mathbf{3 8 0 . 0 0 0}$ francs pour le canton et 700.000 francs pour la Ville) par l'intermédiaire des CEuvres d'entraide.

Le canton reçoit les propositions des différentes organisations privées. La direction des Finances examine alors les différents projets, en fait une sélection qui est présentée ensuite au gouvernement cantonal pour décision. Le coût des projets détermine le montant final de l'aide, qui oscille toujours aux alentours de 300.000 francs. La souplesse du système permet de financer un projet particulièrement intéressant même si son coût est un peu plus élevé que de coutume. Après décision du Conseil d'Etat zurichois et un éventuel examen par la DDA, le montant total de l'aide est alors prélevé sur le bénéfice des loteries et versé aux différentes CEuvres d'entraide.

Pour sa part la Ville veille à disposer toujours d'une quinzaine de propositions de projets, parmi lesquels elle en choisit cinq à six. La sélection se fait essentiellement sur la base des principes de politique de développement examinés plus haut; mais I'on veille scrupuleusement à répartir équitablement les montants disponibles entre toutes les organisations importantes de Suisse. De 1972 à 1981, la commune de Zurich a versé 2,3 millions de francs aux organisations non-confessionnelles, 1,1 million aux œuvres protestantes et 1,4 million à celles liées à l'Eglise catholique. 
Le canton de Genève est un cas un peu particulier dans la mesure où son aide passe par la Fédération genevoise de coopération qui regroupe l'ensemble des groupes et des organisations actives à Genève dans le domaine des relations Suisse-Tiers Monde.

La FGC intervient comme intermédiaire entre ces groupements (dont les principales CEuvres d'entraide nationales), l'Etat, les communes genevoises et la Confédération. Elle prépare et sélectionne les projets que lui soumettent ses membres et les présente ensuite pour accord et financement aux collectivités locales et à l'administration fédérale compétente. Dans ce sens, l'aide des communes et du canton de Genève est à mi-chemin de la coopération "directe» (puisqu'elle passe par une infrastructure spécifique) et de la coopération "indirecte" (puisqu'elle est finalement transférée par le biais de différentes organiations privées et publiques dont les CEuvres d'entraide).

En 1982, trois cantons et une commune ont préféré la formule de I'aide "directe». II s'agit des cantons du Jura, de Bâle, de Vaud et de la commune de Münsingen.

Münsingen est une petite bourgade de la campagne bernoise située entre Berne et Thoune. Fait unique dans les annales de la coopération au développement, elle gère depuis plus de dix ans un projet de développement dans la selva péruvienne.

La charge financière que supporte ainsi la commune politique et les deux Eglises de l'endroit est supérieure aux prestations du canton de Berne et fait de Münsingen I'un des principaux donateurs en matière de coopétation au développement. Si l'on tient compte de la population résidente, la charge par habitant est dix à vingt fois supérieure à celle que l'on rencontre dans les autres communes et cantons. L'originalité de la situation de Münsingen tient aussi à la forme de gestion du projet: un comité formé des représentants de la commune politique et des communautés religieuses prend lui-même toutes les décisions concernant le projet et les transmet à un coopérant employé par la commune qui réside sur place. Depuis plusieurs années Münsingen bénéficie aussi des services de la coopération technique fédérale installés à Lima.

Ce type de formule occasionne des difficultés considérables de gestion et de contrôle. Mais elle présente aussi des avantages non négligeables: une petite enquête menée par un journaliste du "Bund" (10) a démontré que presque toutes les personnes interrogées à Münsingen avaient connaissance du projet "Villa Rica» financé par la commune. L'information et la motivation des citoyens est rendue plus aisée du fait que chacun considère ce projet comme "son" projet.

Le canton de Vaud finance depuis 1972 un projet de formation professionnelle en Tunisie. Le fonctionnaire responsable de la gestion de cette aide évalue les besoins financiers annuels et présente ensuite la facture au Conseil d'Etat vaudois. L'administration cantonale recrute les coopérants envoyés en Tunisie à titre d'enseignants (vingt-quatre au total jusqu'à aujourd'hui). C'est elle aussi qui envoie un responsable sur place à chaque difficulté importante. 
Ce projet de coopération se termine en 1983. Sans être en mesure de se prononcer sur les résultats de ce projet, on peut penser que cette expérience de coopération "directe" aura été marquée par un mode de gestion ne laissant que peu de place à la concertation avec des spécialistes, à l'information et au débat.

La coopération "directe" n'est pas exempte de difficultés, et les collectivités locales sont souvent bien mal armées pour y faire face. A l'Etat de Vaud on ne cache pas qu'un retour à une aide par l'intermédiaire des CEuvres d'entraide n'est pas exclue.

Dans le canton du Jura par contre on tient fermement à la formule d'aide directe. "Peut-être parce que nous venons de débuter», explique C. Jeannerat, responsable du secteur de la coopération au développement dans le Département dirigé par F. Lachat. Jusqu'à fin 1982, le canton du Jura disposait même d'un Délégué à la Coopération, le conseiller aux Etats Roger Schaffter, dont l'une des tâches était l'aide au développement. Récemment une motion Friedli (parti socialiste) a été acceptée par le Parlement jurassien. Le gouvernement cantonal devra veiller désormais à ce que $0,1 \%$ des dépenses du canton soit progressivement consacré à l'aide au développement. En 1983, 125.000 francs ont été budgétés dans le domaine de l'aide au développement; et l'importance de cette somme, qui va aller croissant, est aussi l'un des facteurs qui expliquent l'attachement des Jurassiens à une gestion directe du programme.

D'autres motifs plus curieux expliquent parfois le choix de la coopération directe. Ainsi cette commune où les parlementaires locaux préféreraient ne pas passer par les CEuvres d'entraide qu'ils jugeaient "conservatrices et toutes plus ou moins liées à I'Eglise» !!!

\section{Des exemples originaux}

La solidarité des communes et des cantons ne se limite pas toujours aux formes de l'aide humanitaire ou de la coopération au développement. Certaines collectivités publiques ont fait preuve d'imagination.

L'essentiel de l'aide humanitaire fournie par la Commune de la Chaux-deFonds permet de financer la rééducation d'enfants recueillis par Terre des Hommes. L'hôpital de la ville a aussi été sollicité par les autorités au moment de l'exode massif des réfugiés vietnamiens. Des médecins locaux ont été dépêchés sur le navire "lle de Lumière" en continuant à percevoir leur salaire à I'hôpital. De ce fait, le budget d'aide n'était pas grevé outre mesure.

En 1977, le canton de Schaffhouse a institué un "prix schaffhousois pour la coopération au développement». Ce prix, d'une valeur de 10.000 francs, est attribué chaque année "aux personnes et organisations qui s'efforcent de travailler à la compréhension des problèmes de développement, notamment dans le cadre des relations Suisse-Tiers Monde, ou qui ont contribué à leur solution" (art. 2 de l'Arrêté du Grand Conseil du canton de Schaffhouse sur un "prix schaffhousois pour la coopération au développement" (trad.)(1). 
Les débats à ce propos au Grand Conseil montrent que cette proposition fut amenée par une commission préparatoire du Grand Conseil qui avait à se pencher sur l'éventualité d'une aide cantonale au développement. La commission estimait que, malgré toutes les difficultés financières invoquées, le canton de Schaffhouse ne pouvait pas décemment rester sans rien faire. "Le monde est une unité dont on ne peut pas débarquer" constata le rapporteur de la commission devant le Conseil. En conséquence, la commission préparatoire demanda au Grand Conseil d'inviter le gouvernement cantonal à prendre des dispositions pour que les problèmes de développement soient abordés à tous les niveaux de la scolarité ainsi que dans la formation des adultes. La commission souhaitait aussi que l'administration cantonale et les hautes écoles fassent en sorte que des volontaires puissent être détachés pour mettre leurs compétences personnelles au service de pays en développement. Selon les membres de cette commission, ces mesures et l'institution d'un prix couronnant des efforts faits en faveur de la coopération internationale devaient montrer que le canton de Schaffhouse ne restait pas insensible à la situation des pays en développement et qu'il allait veiller à améliorer la compréhension et l'information des citoyens du canton sur ces problèmes.

Le Conseil d'Etat fut d'un tout autre avis: la santé des finances cantonales ne permettait pas au canton d'améliorer sensiblement sa contribution en faveur de l'aide au développement. Quant à la proposition d'un prix sur ce sujet, le gouvernement cantonal trouvait assez déplacé qu'un canton qui ne faisait pas grand-chose en la matière se permette encore de juger les réalisations des autres.

Le débat en séance plénière fut passablement agité. Le parti démocrate chrétien, les organisations progressistes de Suisse (POCH) et le parti socialiste se rangèrent derrière l'avis de la commission tandis que les radicaux et les xénophobes s'opposèrent à cette proposition comme le Conseil d'Etat. Au vote la proposition de la commission l'emporta par 44 voix contre 18 . Le prix cantonal pour la coopération au développement était né.

Depuis 1978, le prix a été accordé aux Magasins du Monde de Schaffhouse, à la section locale de Terre des Hommes, à l'action "place libre" en faveur des réfugiés du Chili et à deux personnes (dont un médecin) particulièrement méritantes.

\section{LA GESTION DE L'AIDE}

\section{Compétences formelles}

En parcourant les administrations communales et cantonales à la recherche du fonctionnaire responsable de l'aide au développement, on ne tarde pas à être frappé par l'extraordinaire variété des compétences formelles. Diversité fort révélatrice puisqu'en attribuant la gestion de l'aide à l'un ou l'autre secteur de l'administration locale, les autorités dévoilent aussi l'image qu'elles se font elles-mêmes de l'aide au développement. Que la coopération au développement relève du Département des Finances ou du Bureau des Affaires sociales n'est pas tout à fait indifférent; du même coup, c'est tel ou tel aspect de 
I'aide communale ou cantonale qui va être privilégié. Le Département des Finances, par exemple, ne sera pas obsédé par l'information sur le projet. Celui de l'Economie publique sera peut-être tenté d'intégrer certaines de ses priorités en concevant un nouveau projet; il sera en tout cas plus porté à travailler dans la formation professionnelle que dans la santé.

C'est pourquoi il n'est pas inutile, en pénétrant dans une administration publique, d'accorder un regard aux austères plaques grises, à gauche de l'entrée principale, qui vous donneront l'identité du suzerain local de l'aide au développement.

Le canton du Jura a porté la Coopération au niveau suprême puisqu'elle figure déjà dans la dénomination officielle: Département de la Coopération, des Finances et de la Police.

Fidèles à leur image de sérieux, la Ville et le canton de Zurich font tous deux dépendre l'aide au développement de la Direction des Finances.

Le canton de Soleure a confié le dossier au Département de I'Education.

Le canton de Vaud a remis la responsabilité de la gestion du programme de coopération cantonale au Département de l'Agriculture, de l'Industrie et du Commerce.

La diversité n'est pas moindre dans les communes.

A la Chaux-de-Fonds, c'est l'exécutif dans son ensemble qui s'en occupe. La gestion administrative reste dans les mains du chancelier de la Ville.

A Yverdon, c'est la Direction des Affaires sociales qui porte la responsabilité de l'aide communale au développement. Le choix des projets est effectué par la Commission sociale, instance qui, dans le canton de Vaud, est habituellement compétente pour accorder une assistance communale aux personnes qui $n$ 'ont plus d'autres ressources. Les membres de la commission sociale sont nommés en fonction de l'importance des différents courants politiques représentés dans le législatif local.

\section{L'aide et la loi}

Les activités d'une commune ou d'un canton en matière de coopération au développement ou d'aide humanitaire ne rendent pas nécessaire l'existence d'une loi ou d'un règlement. La grande majorité des collectivités publiques se satisfont parfaitement de l'absence de base légale à proprement parler. Cela permet une plus grande souplesse $d^{\prime}$ action et $n$ 'hypothèque pas un avenir financier qui s'annonce incertain.

Le plus souvent les communes ou les cantons se contentent d'associer le législatif local en lui proposant d'inscrire le montant de l'aide au développement au budget annuel. Parfois l'exécutif dispose aussi d'une petite autonomie qui lui permet de prendre des initiatives dans ce domaine, quitte à en référer par la suite aux parlementaires. Lorsque 
ceux-ci souhaitent instaurer une certaine régularité dans l'exercice de I'aide, il leur suffit de voter un arrêté simple, qui donne mandat à l'exécutif d'organiser la gestion concrète des fonds publics affectés.

Pourtant quelques communes et cantons ont été plus loin en ancrant leur aide dans l'appareil légal. Parfois par pur pragmatisme, parfois aussi pour affirmer l'importance que revêt I'aide au développement aux yeux de la communauté en question. Le plus souvent, une base légale devient nécessaire lorsque les promoteurs de l'aide souhaitent la soumettre à des principes de politique de développement (cf. cidessous).

Le canton du Jura est un phénomène tout à fait particulier puisque la coopération est l'une des tâches de l'Etat prévues par la Constitution. Son préambule affirme la volonté du nouveau canton d'encourager la coopération avec les peuples. L'article 53 précise encore: “...l'Etat encourage l'aide humanitaire et coopère au développement des peuples défavorisés."

L'importance de l'aide au développement cantonale étant clairement posée au niveau suprême (ce qui n'est même pas le cas de la Confédération), les Jurassiens $n^{\prime}$ ont pas jugé nécessaire jusqu'ici de la fonder une nouvelle fois dans une loi d'application. Les directives données par le Parlement à l'occasion, telle que la motion Friedli qui fixe le niveau de I'aide cantonale à $0,1 \%$ des dépenses, en tient lieu dans la pratique.

La commune de Zurich a bâti un édifice juridique complet. Le principe d'une coopération au développement dirigée vers l'étranger et vers les régions de Suisse les plus défavorisées a été voté par le peuple et équivaut en quelque sorte à une décision de niveau constitutionnel. Ce principe a ensuite été défini par un arrêté du Conseil communal (législatif). Les derniers détails d'application sont du ressort de l'administration et de règles internes qu'elle s'est fixé.

La mise en place de l'infrastructure juridique zurichoise ne s'est d'ailleurs pas faite sans mal puisque c'est de justesse que le principe de l'aide a été approuvé par le peuple et que cette décision a ensuite été attaquée par un recours administratif: "L'Union des citoyens libres en Suisse et à l'étranger" reprochait au Conseil de Ville d'avoir manipulé la votation en liant l'acceptation de l'aide destinée à des communes suisses à celle dirigée vers les pays en développement. Le recours fut rejeté.

La gestion de l'aide ne dépend pas uniquement du cadre légal en vigueur. Plus proches de la population que la Confédération, les communes et leurs organes politiques peuvent jouer un rôle non négligeable dans la gestion des deniers publics voués à l'aide au développement. $D^{\prime}$ autant plus que les responsables politiques locaux ont souvent un accès plus direct à l'administration de leur commune que cela n'est possible au niveau de l'Etat central. L'implication des organes politiques et administratifs est donc un élément d'importance.

La motion Deluz, déposée au Conseil communal d'Yverdon, avait notamment pour objectif d'engager au maximum les députés du législatif yverdonnois sur 
le terrain de la coopération au développement en les associant à la gestion de l'aide. Le motionnaire désirait notamment qu'une commission ad hoc soit constituée et que cette derniere propose chaque année un ou plusieurs projets au Conseil communal. Par cette formule, F. Deluz entendait introduire le débat de politique de développement jusque dans les travées du législatif municipal et espérait ainsi «donner une plus large audience au projet présenté que si la décision n'apparaissait que sous forme d'un compte inscrit au budget par la Municipalité" (12). Cette dernière préféra pourtant des méthodes plus orthodoxes et confia la responsabilité de la gestion de l'aide à la Commission sociale déjà existante.

\section{La collaboration des communes}

Lorsqu'un poste "aide au développement" apparaît à l'horizon budgétaire d'une commune, il n'est pas rare d'entendre les opposants crier à l'inefficacité d'une aide qui ne peut être que trop minime ou dont la gestion occupe des forces disproportionnées.

Sur ce dernier point, les fonctionnaires interrogés avouent ne passer qu'une partie de leur temps de travail sur le dossier de l'aide au développement. La Ville de Zurich, dont la contribution est la plus importante de toutes les communes de Suisse, ne consacre que 20 à $25 \%$ d'un poste de fonctionnaire à cette tâche. Le taux est identique dans le canton du Jura dont le budget $d$ 'aide est plus modeste mais qui gère directement son programme de coopération aux Seychelles.

Quant au volume prétendument trop réduit de l'aide des communes, des parades originales ont aussi été trouvées: certaines communes travaillent main dans la main à la gestion d'un même projet. Elles peuvent ainsi financer l'essentiel du projet et se partager les tâches de gestion et de contrôle.

Depuis 1982, la Commune d'Yverdon et l'Association de coopération technique de Nyon et environ (qui bénéficie aussi de l'appui financier de quelques communes de la région nyonnaise) ont uni leurs efforts pour prendre en charge le soutien à l'hôpital d'Aïn Draham (Tunisie).

Les villes de Saint-Gall, Winterthour et Schaffhouse collaborent depuis plusieurs années au financement d'un projet de développement en Colombie. Par l'intermédiaire de Swissaid, les trois communes ont permis à des coopératives paysannes d'acquérir des moyens de transport, de construire de nouveaux locaux et d'inaugurer un fonds de roulement pour la commercialisation du riz, des haricots et de la canne à sucre. Les trois villes ont aussi œuvré au Gabon, au Sri Lanka, en Haute-Volta. Elles assument à tour de rôle chaque année la gestion et le contrôle du projet financé par les trois communes. Leur objectif est de se répartir ainsi les charges tout en se donnant les moyens de soutenir un projet suffisamment important. 


\section{L'INFORMATION: PIERRE D'ANGLE DE LA POLITIQUE DE DEVELOPPEMENT}

II ne fait pas de doute que l'information du public est une des clés de I'aide au développement communale et cantonale. L'aide humanitaire et surtout la coopération au développement sont des tâches relativement nouvelles pour la collectivité, à quelque niveau que ce soit. La réalisation de programmes de développement dans le Tiers Monde est une tâche ingrate et de longue haleine. Loin des yeux, loin du cœur dit le proverbe. Cela est vrai aussi pour la coopération, dont les réalisations n'ont pas l'avantage d'apparaître concrètement sous les yeux des contribuables. Ses bénéficiaires $n^{\prime}$ ont le plus souvent pas les moyens de se faire entendre sous les fenêtres du Palais fédéral lorsque le gouvernement tente d'économiser quelques sous sur les crédits d'assistance.

La situation n'est pas meilleure sur la scène politique des communes et des cantons. Les responsables politiques locaux, conscients de l'importance du problème, n'ont pas hésité jusqu'à aujourd"hui à se faire les avocats d'une aide au développement locale, même modeste. Mais les temps ont changé et la situation financière des communes et des cantons n'est plus celle du début des années septante. La crise économique qui frappe certaines d'entre elles représente le principal danger pour les années à venir. L'aide au développement, parent pauvre du budget local, risque bien de faire les frais d'éventuelles restrictions financières. A tout le moins, son extension est menacée.

L'importance de l'enjeu démontre à lui seul que toutes les forces seront nécessaires pour convaincre l'opinion publique d'accorder une attention accrue à la solidarité internationale. Les communes et les cantons ont un rôle irremplaçable à jouer sur ce plan. Les contacts étroits que les communes entretiennent avec leurs administrés favorisent beaucoup le passage d'une information liée aux activités d'aide au développement.

Malgré ses moyens financiers relativement importants, la Confédération mettra bien du temps à combler la distance qui la sépare de ses administrés dans ce domaine si elle n'est pas épaulée par les communes et les cantons. L'administration fédérale l'a d'ailleurs bien compris puisqu'elle encourage les communes à œuvrer avec elle à la coopération internationale dans l'espoir de favoriser ainsi son enracinement dans la population.

Au-delà de la sensibilistion des citoyens, l'information assurée par les collectivités locales a aussi pour fonction de permettre à tout un chacun de contrôler l'usage qui est fait des fonds publics. 
La commune de Zurich a fêté en 1982 ses dix ans d'activités dans le secteur de la coopération au développement. A cette occasion elle a organisé une exposition présentant les projets qui avaient été soutenus au cours de la décennie. De très nombreuses photos illustraient les réalisations de projets en Asie, en Afrique et en Amérique latine. Des montages audio-visuels permettaient aux classes de passage $d^{\prime}$ aborder quelques-uns des problèmes rencontrés par les pays en développement. Pour les adultes, un cycle de films provenant de pays du Tiers Monde complétaient l'exposition.

Soucieuse de ne pas prêter le flanc à la critique, la Ville a financé l'exposition par un prélèvement sur le fonds des héritages non réclamés.

Certaines communes ont tenté de dépasser le cadre de la coopération au développement pour aborder plus largement la problématique du sous- ou mal-développement.

La commune genevoise de Meyrin a fait de très importants efforts pour mettre sur pied, en 1981, une semaine d'animation et d'information sur le Tiers Monde qui se tenait dans les locaux communaux au centre de la ville. Tous les groupes de l'endroit engagés en faveur de la solidarité avec les peuples du Tiers Monde ont été invités à présenter leurs activités.

Des représentants des CEuvres d'entraide ont pu répondre aux questions de participants lors de forums. L'animation culturelle (danses, films, etc...) a permis aux habitants de Meyrin, aux écoles notamment, de goûter pendant quelques jours à un climat peu traditionnel pour la banlieue genevoise.

L'idée a d'ailleurs été reprise puisqu'en septembre 1983, la commune voisine de Lancy (elle aussi très active dans l'aide au développement) a préparé une manifestation du même genre.

La Ville de Berne a entrepris, parallèlement à ses efforts de coopération et d'aide humanitaire, de financer en partie les activités du service Ecole Tiers Monde, un organisme dépendant de la Communauté de travail des CEuvres d'entraide, dont la tâche est de collaborer avec les administrations de l'enseignement public et avec les enseignants de manière à améliorer la quantité et la qualité du matériel pédagogique concernant les pays en développement.

Le canton de Schaffhouse représente un cas extrême en la matière puisque l'ensemble de ses efforts porte sur l'information. Aucun projet de développement $n$ 'est soutenu par le canton en 1982, mais celui-ci finance, en revanche, depuis 1978, le "prix de la coopération au développement" par un montant annuel de 10.000 francs dans le but de sensibiliser la population du canton aux problèmes rencontrés par les pays en développement et aux efforts entrepris pour tenter $d^{\prime} y$ apporter une solution (cf. ci-dessus).

\section{CONCLUSION}

La palette de l'aide communale et cantonale au développement présente une formidable diversité. De la commune qui verse 1.000 francs pour les bonnes œuvres à celle qui gère elle-même un projet en Amérique latine, la panoplie est presque complète. 
Cet aspect hétéroclite est toute la richesse de l'aide locale. Au moment $d^{\prime}$ 'entreprendre de nouveaux efforts, au moment $d$ 'inaugurer un budget spécial pour l'aide au développement, chaque commune et chaque canton réinvente la coopération au développement. Nous avons constaté en effectuant cette petite enquête que la plupart des responsables communaux et cantonaux n'avaient pas connaissance des expériences faites par leurs collègues ailleurs en Suisse. Non pas qu'ils aient été bornés au point de rester obnubilés par le monde clos de leur administration, mais parce que le plus souvent c'est sur la scène communale ou cantonale que se jouait véritablement le profil du projet.

Dans chaque cas les obstacles sont différents et les moyens de les surmonter aussi. Ici, les adversaires sont des xénophobes endurcis pour qui tout argent qui passe les frontières helvétiques ne peut qu'être gaspillé. Là, par contre, des préjugés d'une autre époque entachent l'activité des organisations privées. Ailleurs encore un mouvement politique hostile à la coopération au développement juge "qu'il y a bien assez à faire en Suisse sans que I'on doive chercher au bout du monde».

A l'opposé, chaque localité compte aussi de multiples facteurs favorables à l'existence ou à la croissance de l'aide au développement. Ici, c'est le maire dont la fille séjourne dans une grande ville africaine qui décide de "faire quelque chose", là, c'est l'Eglise locale ou tout un mouvement politique qui prend les choses en mains avec des objectifs précis.

Tous ces éléments doivent prendre place dans le projet de la commune ou du canton. D'où le paysage contrasté que l'on découvre en passant d'une coopération communale à l'autre.

Mais cette diversité n'est pas synonyme d'inefficacité. "Que sont les 7 millions de l'aide communale et cantonale face au demi-milliard dépensé chaque année par la Confédération?" dira-t-on avec condescendance. Bien peu de chose en effet si l'on s'en tient à l'aspect financier du problème. Mais tout ou presque tout si l'on considère la richesse de toutes ces expériences. Chacune de ces petites collectivités doit convaincre sa population et sa classe politique de la nécessité de I'aide. Plus qu'au Parlement fédéral, c'est dans les communes d'abord que l'aide au développement passe l'examen d'entrée. C'est là que se font et que se défont les préjugés qui détermineront l'attitude de nos concitoyens vis-à-vis de l'aide au développement et du nouveau style que la Suisse devra imprimer à ses relations avec les nations du Tiers Monde. Dans le domaine de l'information, les cantons et les communes ont sans conteste un rôle primordial à jouer. Au moment où la crise aggrave le fossé qui sépare le Nord et le Sud, la solidarité est plus indispensable que jamais. Ne faut-il pas tout faire pour maintenir I'ouverture de la Suisse sur le monde? Et abandonner une fois pour toutes l'illusion du "Sonderfall Schweiz». 


\section{Notes}

1. Swissaid, Aide suisse aux pays en développement, Berne, annuel.

2. OCDE, Rapport du Comité d'aide au développement, Paris, annuel.

3. Swissaid, Aide suisse aux pays en développement 1981, Berne, 1982, p. 2.

4. Cf. à ce propos G. Rist, Le rôle des organisations privées suisses de développement, in Annuaire Suisse-Tiers Monde, IUED, Genève, 1982.

5. Message du Conseil fédéral sur la Loi fédérale sur la Coopération au développement et l'aide humanitaire, FF 1973 I 886.

6. FF 1973 । 886.

7. Cf. P.F. Chatton, L'affront des Jurassiens, in L'Hebdo, Lausanne, 7 juillet 1983.

8. Auszug aus dem Protokoll des Stadtrates von Zürich, vom 5. März 1975649/2. Grundsätze bezüglich des Empfängerlandes.

9. Finanzamt der Stadt Zürich, 1972-1981 Entwicklungshilfe der Stadt Zürich im In- und Ausland, Zürich 1982, p. 7, Allgemeine Voraussetzungen.

10. "Münsingen und die Dritte Welt, ein ganzes Dorf steht hinter einem Entwicklungsprojekt in Peru», in Der Bund, Bern, 23. März 1983.

11. Beschluss des Grossen Rates des Kantons Schaffhausen über einen "Schauffhauser Preis für Entwicklungszusammenarbeit» vom 29. August 1977.

12. Municipalité d'Yverdon, Rapport sur la motion F. Deluz - Aide au Tiers Monde, du 8 mai 1980. 


\begin{tabular}{|c|c|c|c|c|c|}
\hline $\begin{array}{l}\text { Contributions } \\
\text { communales } \\
\text { par canton }\end{array}$ & $\begin{array}{l}\text { Coopération } \\
\text { au dévelop- } \\
\text { pement } 1 \text { ) }\end{array}$ & $\begin{array}{l}\text { Aide } \\
\text { humanitaire } \\
\text { 1) }\end{array}$ & Total & $\begin{array}{l}\text { Population } \\
\text { résidante des } \\
\text { communes2) }\end{array}$ & $\begin{array}{l}\text { Contributions } \\
\text { par habitant } \\
\text { en Sfr. }\end{array}$ \\
\hline
\end{tabular}

\section{Aargau AG}

$\begin{array}{lr}\text { Aarau } & 19 \\ \text { Baden } & 10 \\ \text { Ennetbaden } & 3 \\ \text { Mellingen } & 3 \\ \text { Reinach } & 3\end{array}$

Rheinfelden

Spreitenbach 20

Stetten 1

Untersiggenthal 8

Wettingen 8

Neuenhof 2

19

0

3

3

3

0

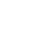

8

9

2

77

10

87

\section{Appenzell AR}

Heiden

3620

0,55

\section{Basel-Land BL}

Aesch

Allschwil

Augst

Arlesheim

18

1

5

Binningen

24

Frenkendorf

5

Münchenstein $\quad 30$

Reinach
2

$\begin{array}{rrr}2 & 7954 & 0,25 \\ 18 & 17952 & 1,00 \\ 1 & 836 & 1,20 \\ 5 & 8224 & 0,60 \\ 24 & 14195 & 1,69 \\ 5 & 5438 & 0,92 \\ 30 & 11002 & 2,73 \\ 40 & 17813 & 2,25\end{array}$

1,20

0,72

1,15

0,91

0,53

0,11

2,78

1,04

1,74

0,93

0,28

$7203 \quad 0,28$

- 


\begin{tabular}{|c|c|c|c|c|c|}
\hline $\begin{array}{l}\text { Contributions } \\
\text { communales } \\
\text { par canton }\end{array}$ & $\begin{array}{l}\text { Coopération } \\
\text { au dévelop- } \\
\text { pement }\end{array}$ & $\begin{array}{l}\text { Aide } \\
\text { humanitaire }\end{array}$ & Total & $\begin{array}{l}\text { Population } \\
\text { résidante des } \\
\text { communes }\end{array}$ & $\begin{array}{l}\text { Contributions } \\
\text { par habitant } \\
\text { en Sfr. }\end{array}$ \\
\hline
\end{tabular}

\section{Basel-Stadt BS}

\begin{tabular}{|c|c|c|c|c|c|}
\hline Riehen & 30 & 10 & 40 & 20611 & 1,9 \\
\hline \multicolumn{6}{|l|}{ Bern BE } \\
\hline Bern & 200 & 100 & 300 & 145254 & 2,07 \\
\hline Bienne & 24 & 16 & 40 & 53793 & 0,74 \\
\hline Bolligen & 50 & & 50 & 32312 & 1,55 \\
\hline Bremgarten & 10 & & 10 & 3355 & 2,98 \\
\hline Burgdorf & 1 & & 1 & 15379 & 0,07 \\
\hline Interlaken & 3 & & 3 & 4852 & 0,62 \\
\hline Köniz & 14 & 4 & 18 & 33441 & 0,54 \\
\hline Laufen & 10 & & 10 & 4444 & 2,25 \\
\hline Lyss & 10 & & 10 & 8723 & 1,15 \\
\hline Münsingen & 64 & 2 & 66 & 9340 & 7,07 \\
\hline Muri b.Bern & 10 & & 10 & 12285 & 0,81 \\
\hline Spiez & 5. & 3 & 8 & 9800 & 0,82 \\
\hline Steffisburg & 25 & & 25 & 12539 & 1,99 \\
\hline Thun & & 9 & 9 & 36891 & 0,24 \\
\hline Wahlern & 4 & & 4 & 5104 & 0,78 \\
\hline Worb & 5 & & 5 & 11080 & 0,45 \\
\hline Zollikofen & 40 & & 40 & 8717 & 4,59 \\
\hline
\end{tabular}

\section{Genève GE}

1
6
56
50
13
5
3
20
352
12

Aire-La-Ville
Anières
Bardonnex
Bernex
Carouge
Cartigny
Chêne-Bougeries
Choulex
Collex-Bossy
Collonge-
Bellerive
Corsier
Dardagny
Genève
Genthod

Aire-La-Ville

Bardonnex 6

Bernex 56

Carouge 50

Chêne-Bougeries

Choulex

Collonge-

Bellerive

Corsier

Genève

12

\section{, 94}

2,07
0,74

1,55

2,98

0,62

0,54

2,25

7,07

0,81

1,99

0,24

0,78

4,59

\section{$34 \quad 609$}

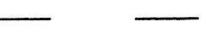

$\begin{array}{rrrr} & 1 & 406 & 2,46 \\ 2 & 2 & 1295 & 1,54 \\ 2 & 8 & 1553 & 5,15 \\ 1 & 57 & 8055 & 7,08 \\ & 50 & 13100 & 3,82 \\ & 13 & 507 & 25,64 \\ 10 & 10 & 9068 & 1,10 \\ & 5 & 698 & 7,16 \\ & 3 & 524 & 5,73 \\ & & & \\ 10 & 30 & 4531 & 6,62 \\ 1 & 1 & 1302 & 0,77 \\ 2 & 2 & 678 & 2,95 \\ 30 & 382 & 156505 & 2,44 \\ & 12 & 1175 & 10,21\end{array}$




\begin{tabular}{|c|c|c|c|c|c|}
\hline $\begin{array}{l}\text { Contributions } \\
\text { communales } \\
\text { par canton }\end{array}$ & $\begin{array}{l}\text { Coopération } \\
\text { au dévelop- } \\
\text { pement }\end{array}$ & $\begin{array}{l}\text { Aide } \\
\text { humanitaire }\end{array}$ & Total & $\begin{array}{l}\text { Population } \\
\text { résidante des } \\
\text { communes }\end{array}$ & $\begin{array}{l}\text { Contributions } \\
\text { par habitant } \\
\text { en Sfr. }\end{array}$ \\
\hline
\end{tabular}

Grand-

Saconnex

Laconnex

Lancy

Meinier

Meyrin

Onex

10
175
2
100
4

Perly-Certoux

3

Plan-Les-Ouates

3

Pregny-Chambesy 8

Thõnex

Troinex

3

Vernier

19

3

10

3

6333

1,58

384

7,81

2

$177 \quad 23527$

7,52

$2 \quad 1390$

1,44

20

120

18808

6,38

5

$9 \quad 16956$

0,53

$3 \quad 2232$

1,34

$3 \quad 3647$

0,82

$8 \quad 1939$

4,13

9241

1,73

$\begin{array}{llll}8 & 11 & 1 & 161\end{array}$

9,47

21

27962

0,75

842

117

959

\section{Graubünden GR}

Vaz

6

6

2067

2,90

Jura JU

Delémont

Porrentruy
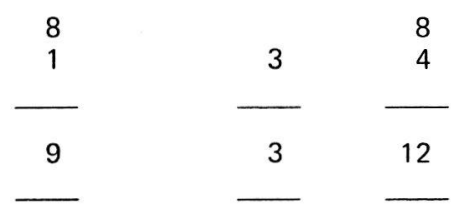

\section{Luzern LU}

Ballwil

Buttisholz

Littau

Kriens

\begin{tabular}{rrr}
5 & 13 & 18 \\
2 & & 2 \\
20 & 1 & 1 \\
& & 20 \\
27 & 14 & 41 \\
\hline
\end{tabular}

0,25

0,57 
Contributions communales par canton
Coopération au développement
Aide humanitaire
Total

Population Contributions résidante des par habitant communes en Sfr.

\section{Neuchâtel NE}

La Chaux-de-Fonds

Le Locle

2
20

41

41

37234

1,10

Neuchâtel

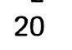

4

2
24

12039

0,17

34428

0,70

\section{Obwalden OW}

\begin{tabular}{lll}
-22 & - & - \\
- & 45 & 67 \\
\hline
\end{tabular}

Sarnen

7372

0,14

\section{St. Gallen SG}

Jona

5

Oberriet

Rorschach

6

St Gallen

25

5

12156

0,41

6222

0,16

9878

0,61

75847

0,40

\section{Schaffhausen SH}

Schaffhausen

8

33

34250

0,96

\section{Solothurn SO}

Bellach

Biberist

Dornach

Dulliken

Etziken

Grenchen

34

Langendorf

Luterbach

Nunningen

Olten

2

1

20

Riedholz

1

Trimbach

Zuchwil

15

3

7519

0,66

5442

0,55

3

4465

1,12

666

3,00

16800

2,20

3124

1,28

2880

0,69

1472

0,68

3

18991

1,21

1398

0,72

7065

2,12

8223

0,85 


$\begin{array}{lllll}\begin{array}{l}\text { Contributions } \\ \text { communales } \\ \text { par canton }\end{array} & \begin{array}{l}\text { Coopération } \\ \text { au dévelop- } \\ \text { pement }\end{array} & \begin{array}{l}\text { Aide } \\ \text { humanitaire }\end{array} & \begin{array}{l}\text { Total } \\ \text { Population }\end{array} \begin{array}{l}\text { Contributions } \\ \text { résidante des } \\ \text { par habitant } \\ \text { communes }\end{array} \text { en Sfr. }\end{array}$

\section{Tessin TI}

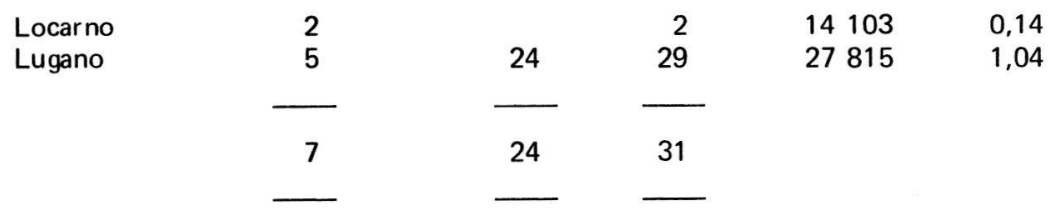

Thurgau TG

Frauenfeld

10

10

18607

0,54

Vaud VD

Aigle
Aubonne
Corseaux
Epalinges
Jouxtens
Lausanne
Montreux
Le Mont-sur
Lausanne
Nyon
Pully
St. Sulpice
Vevey

1

4

1

1

19

1

20

2

6233

0,32

1

1958

0,51

$6 \quad 1901$

3,16

5160

0,19

516

1,94

1

127349

0,31

21

19685

1,12

22

3664

0,27

12842

0,16

14988

2,27

2129

0,47

16139

3,59

43

15

58

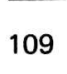

59

\section{8}

\section{Valais VS}

Brig

Monthey

Sion

5
6
24

24

35

35

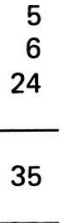

\section{Zug ZG}

Unterägeri

Zug

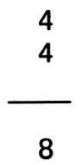

\begin{tabular}{rrr}
4 & 5371 & 0,74 \\
4 & 21609 & 0,19 \\
& & \\
\hline 8 & &
\end{tabular}




\begin{tabular}{lllll}
$\begin{array}{l}\text { Contributions } \\
\text { communales } \\
\text { par canton }\end{array}$ & $\begin{array}{l}\text { Coopération } \\
\text { au dévelop- } \\
\text { pement }\end{array}$ & $\begin{array}{l}\text { Aide } \\
\text { humanitaire }\end{array}$ & $\begin{array}{l}\text { Total } \\
\text { résidante des } \\
\text { communes }\end{array}$ & $\begin{array}{l}\text { Contributions } \\
\text { par habitant } \\
\text { en SFr. }\end{array}$ \\
\hline
\end{tabular}

\section{Zürich ZH}

\begin{tabular}{|c|c|c|c|c|c|}
\hline Adliswil & 20 & & 20 & 16418 & 1,22 \\
\hline Bülach & 62 & & 62 & 12292 & 5,04 \\
\hline Dübendorf & 10 & & 10 & 20683 & 0,48 \\
\hline Erlenbach & 20 & & 20 & 4331 & 4,62 \\
\hline Gossau & 5 & & 5 & 6205 & 0,80 \\
\hline Hinwil & 27 & & 27 & 7554 & 3,57 \\
\hline Illnau-Effretikon & 8 & & 8 & 14788 & 0,54 \\
\hline Kloten & 116 & & 116 & 15845 & 7,32 \\
\hline Küsnacht & 215 & 40 & 255 & 12766 & 19,97 \\
\hline Maur & 50 & & 50 & 6222 & 8,04 \\
\hline Meilen & 45 & & 45 & 10430 & 4,31 \\
\hline Rüschlikon & 25 & & 25 & 4778 & 5,23 \\
\hline Rüti & 8 & 1 & 9 & 9331 & 0,96 \\
\hline Schlieren & 5 & & 5 & 12891 & 0,39 \\
\hline Uster & 8 & & 8 & 23702 & 0,34 \\
\hline Wallisellen & 1 & 25 & 26 & 10887 & 2,39 \\
\hline Wetzikon & 1 & & 1 & 15859 & 0,06 \\
\hline Winterthur & 80 & & 80 & 86758 & 0,92 \\
\hline Zollikon & 210 & 20 & 230 & 12134 & 18,96 \\
\hline Zumikon & 20 & & 20 & 4408 & 4,54 \\
\hline \multirow[t]{2}{*}{ Zürich } & 700 & 80 & 780 & 369522 & 2,11 \\
\hline & 1636 & 166 & 1802 & & \\
\hline
\end{tabular}

TO T A L

3583

$603 \quad 4186$

\section{RESUME}

Contributions

cantonales

$\begin{array}{lll}2318 & 1012 & 3330\end{array}$

Contributions

communales

\begin{tabular}{lll}
3583 & 603 & 4186 \\
\hline 5901 & - & -
\end{tabular}

